\title{
Genetic Encoding of Cyanopyridylalanine for In-Cell Protein Macrocyclization by the Nitrile-Aminothiol Click Reaction
}

\author{
Elwy H. Abdelkader, ${ }^{[b] \dagger}$ Haocheng Qianzhu, ${ }^{[a]+}$ Josemon George, ${ }^{[a]}$ Rebecca L. Frkic, ${ }^{[b]}$ Colin J. \\ Jackson, ${ }^{[b]}$ Christoph Nitsche, ${ }^{[a]}$ Gottfried Otting, ${ }^{[b]}$ and Thomas Huber ${ }^{*[a]}$
}

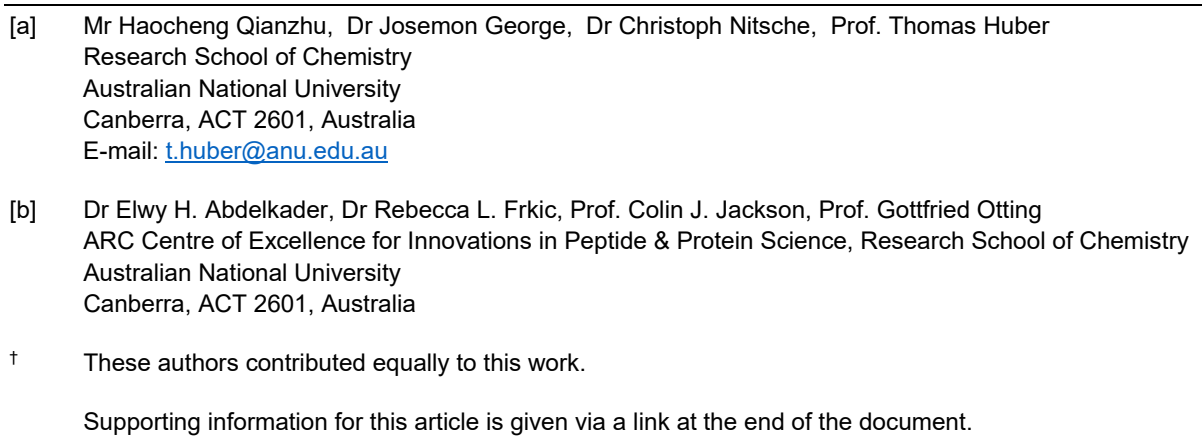

\begin{abstract}
Cyanopyridylalanines are non-canonical amino acids that react with aminothiol compounds under physiological conditions in a biocompatible manner without requiring added catalyst. Here we present newly developed aminoacyl-tRNA synthetases for genetic encoding of meta- and para-cyanopyridylalanine to enable the sitespecific attachment of a wide range of different functionalities. The outstanding utility of the cyanopyridine moiety is demonstrated by examples of (i) post-translational functionalization of proteins, (ii) incell macrocyclization of peptides and proteins, and (iii) protein stapling. The biocompatible nature of the protein ligation chemistry enabled by the cyanopyridylalanine amino acid opens a new path to specific in vivo protein modifications in complex biological environments.
\end{abstract}

Bioorthogonal reactions for site-specific protein conjugation with chemical and biochemical tags have a wide range of applications in the material, biological, and health sciences. ${ }^{[1]}$ The siteselective modification of a target protein can be used to confer specific biophysical properties ${ }^{[2]}$ and install labels for spectroscopic imaging and tracking of proteins by fluorescence, ${ }^{[3]}$ nuclear magnetic resonance, ${ }^{[4]}$ or electron paramagnetic resonance spectroscopy techniques. ${ }^{[5]}$

Site-specific incorporation of noncanonical amino acids (ncAAs) by genetic encoding gives precise control of the sites, where new functional groups are installed in a target protein, ${ }^{[6]}$ but there is only a very small number of established bioorthogonal reactions. To date, the most prominent examples are coppercatalysed azide-alkyne cycloadditions (CuAAC) and inverseelectron demand Diels-Alder reactions (IEDDA). ${ }^{[7]}$ Copper catalysts are barely compatible with physiological conditions, and strain promoted cycloaddition and IEDDA reactions add relatively large non-biological chemical moieties, the synthesis of which can be challenging. In the light of recent progress in genetic code reprogramming to incorporate multiple, distinct ncAAs with different bioorthogonal functionalities into a single protein, ${ }^{[8]}$ there is an unmet demand for additional genetically encoded ncAAs that enable stable conjugations in a reaction that is not only biocompatible but fundamentally different from established bioorthogonal reactions.

The nitrile-aminothiol (NAT) click reaction is a condensation reaction between electrophilic nitriles and 1,2-aminothiols, which proceeds rapidly under biological conditions without the need of any added catalyst and has been shown to be compatible with all canonical amino acids within peptides, except cysteine at the $\mathrm{N}$ terminus of a polypeptide chain. ${ }^{[9]}$ The present work demonstrates its suitability for in vivo protein modification.

To enable site-specific NAT click reactions on proteins, we first identified pyrrolysyl-tRNA synthetase (PyIRS) mutants specific for meta- and para-cyanopyridylalanines (mCNP and pCNP, Figure 1). The synthetases were selected from a library of PyIRS mutants derived from the methanogenic archaeon ISO4G1 (G1PyIRS), using our previously reported screening approach based on fluorescence-activated cell sorting (FACS), ${ }^{[10]}$ and enabled site-specific incorporation of these amino acids in response to an amber stop codon.

In the absence of a crystal structure for G1PyIRS, mutation sites were chosen based on the amino acid sequence alignment between G1PyIRS and Methanosarcina mazei pyrrolysyl-tRNA synthetase (MmPyIRS) (Figure S1). By examining the crystal structure of $M m$ PyIRS (PBD ID: 2Q7E), ${ }^{[1]}$ seven residues in MmPyIRS (L305, Y306, N346, C348, Y384, V401, W417) were hypothesized to influence substrate recognition (Figure S2) and the corresponding residues were randomized in the G1PyIRS library (Supplementary Methods). 
a

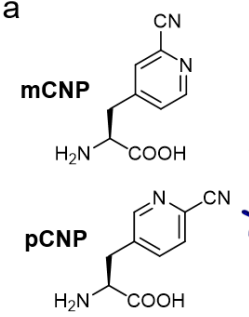

C

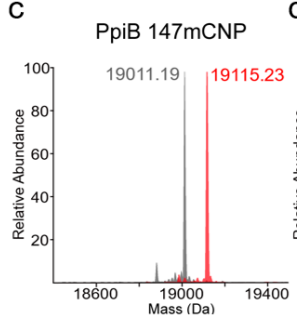

d

b
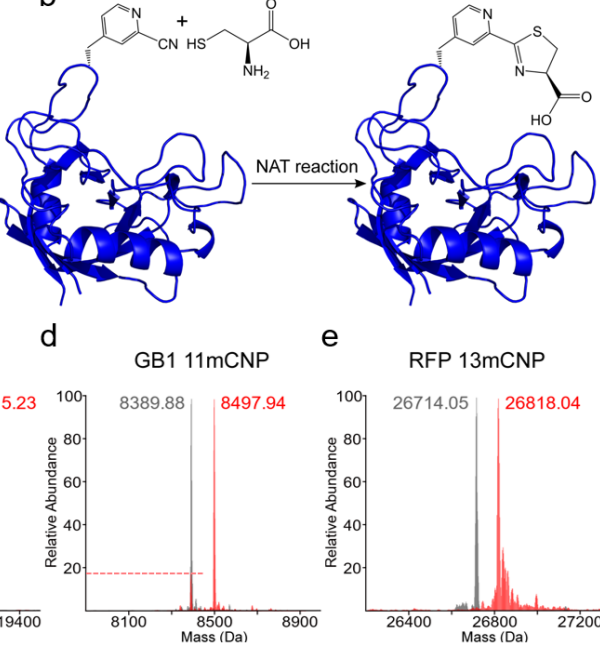

e

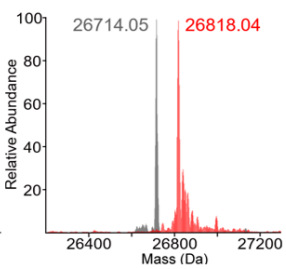

Figure 1. Structures and applications of the cyanopyridylalanines $\mathrm{mCNP}$ and pCNP. a, Chemical structures of mCNP and pCNP. b, Example of a NAT reaction between genetically incorporated $\mathrm{mCNP}$ and cysteine, which proceeds in aqueous solution at neutral $\mathrm{pH}$ and ambient temperature. c-e, Intact protein mass spectrometry analysis of the reaction between genetically incorporated $\mathrm{mCNP}$ and cysteine. The grey and red spectra are of samples before and after the NAT reaction, respectively, and the red dashed line indicates the level of unreacted protein educt. Expected masses are reported in Table S4.

The plasmid library encoding G1PyIRS mutants and the orthogonal ${ }^{\text {G1PyltRNA }}$ CUA (pBK-G1RS) was co-transformed into $E$. coli DH10B cells with the selection plasmid pBAD-H6RFP, which contains the mCherry red fluorescent protein (RFP) gene preceded by a $\mathrm{His}_{6}$ tag with an amber stop codon at position 8 (His6-TAG-RFP). The transformed cells were subjected to multiple rounds of selection under positive (in the presence of the target ncAA) or negative (without the target ncAA) growth conditions. After each selection round, the FACS results showed clear enrichment of the target population. Cells containing synthetase candidates collected from the final round of selection were characterized individually (Figures S3 and S4, and Tables S1 and S2).

The best G1PyIRS mutant for mCNP incorporation (G1mCNP34, in the following referred to as G1mCNPRS) featured the mutations L124A, Y125L, V167A, Y204W, and A221S. Despite randomization in the library, N165 and W237 were the same as in the wild-type sequence. The G1PyIRS mutant for site-specific incorporation of pCNP (G1pCNP37, in the following referred to as G1pCNPRS) carries the mutations L124A, Y125F, Y204W, A221S, and W237Y, with N165 and V167 remaining unchanged. To gain an atomic level understanding of the mutations, we crystallized G1mCNPRS and solved its structure at a resolution of 2.2 $\AA$ (PDB ID: 7R6O; Figure S5; Table S8). As expected, the structure of the amino acid binding domain of the G1mCNP tRNA synthetase is highly conserved, but shows differences in in the loop connecting $\beta$-strands 5 and 6 , which contains Y204W as one of the key residues of the substrate binding site.
To produce proteins with cyanopyridylalanine residues in high yield, we used our previously developed two-plasmid system for in vivo incorporation of ncAAs via amber stop codon suppression. ${ }^{10 \mathrm{~b}]}$ The gene of G1mCNPRS or G1pCNPRS, together with the orthogonal ${ }^{G 1 P y}{ }_{1} R N A_{C U A}$, was cloned into a highcopy number pRSF plasmid to obtain the plasmids pRSFG1mCNPRS and pRSF-G1pCNPRS, respectively, while the gene for the protein of interest was cloned into a low-copy number pCDF plasmid. Using the pCDF/pRSF system, mCNP and pCNP were incorporated with high fidelity and yield, for both single- and double-amber mutants, without any evidence of adduct formation with intracellular metabolites (Figure S6, Table S3). In addition, neither mCNP nor pCNP had any negative effect on $E$. coli cell growth (Figure S7). Next, we tested the reactivity of the incorporated cyanopyridylalanines in the NAT click reaction using L-cysteine as a model 1,2-aminothiol (Figure 1b). Different proteins containing $\mathrm{mCNP}$ or pCNP were incubated with $5 \mathrm{mM}$ cysteine at $25^{\circ} \mathrm{C}$. Monitoring the reaction by intact protein mass spectrometry showed formation of the thiazoline product in greater than $90 \%$ yield after $4 \mathrm{~h}$ (Figure 1c-e), in agreement with the reaction kinetics reported previously for peptides. ${ }^{[9 b]}$ The reaction was not impeded by the presence of $10 \mathrm{mM}$ (tris(2carboxyethyl)phosphine (TCEP) added to prevent oxidation of thiol groups during the reaction.

We previously reported that mCNP installed in peptides by solid-phase peptide synthesis readily undergoes spontaneous cyclization with an $\mathrm{N}$-terminal cysteine residue in aqueous buffers at $\mathrm{pH} 7.5 .^{[9 \mathrm{a}]}$ To test the viability of the cyclization reaction in a protein, we installed $\mathrm{mCNP}$ in the fusion protein NT-Ubi 7X (X indicating the position of the ncAA in the amino acid sequence), which comprised an N-terminal NT solubility tag ${ }^{[13]}$ followed by a short linker containing the modified TEV protease recognition sequence ENLYFQC and human ubiquitin ${ }^{[14]}$ at the C-terminal end (Figure 2a). ${ }^{[15]}$ The fusion protein was readily expressed in $E$. coli and purified using Ni/nitrilotriacetic acid (Ni-NTA) resin. 256 and $162 \mathrm{mg}$ of purified protein were obtained per $1 \mathrm{~L}$ cell culture with $\mathrm{mCNP}$ or $\mathrm{pCNP}$, respectively, with high incorporation yield as indicated by intact protein mass spectrometry (Figure S8). The cyclization reaction was triggered by digestion with TEV protease to expose the cysteine residue of the TEV recognition sequence at the $\mathrm{N}$-terminus. The cleaved protein product Cys-Ubi $7 \mathrm{X}$ cyclized within 15 minutes, as indicated by mass spectrometry. The TEV protease cleavage was complete after 4 hours, resulting in more than $75 \%$ cyclized Cys-Ubi $7 X$ (Figure 2c and d).

Encouraged by these results, we examined the utility of the intramolecular NAT click reaction for in vivo peptide cyclization. To generate the required $\mathrm{N}$-terminal cysteine residue, the protein needs to be cleaved inside the bacterial cell. This was achieved by a 3-plasmid system comprising a pCDF plasmid for the expression of NT-Ubi 7X, the pRSF plasmid containing the orthogonal PyIRS system, and a pBAD-TEV plasmid for coexpression of TEV protease. The results showed that Cys-Ubi $7 X$ was produced in high yield (134 $\mathrm{mg}$ and $60 \mathrm{mg}$ of purified protein per $1 \mathrm{~L}$ cell culture with $\mathrm{mCNP}$ or $\mathrm{pCNP}$, respectively) and either sample was found to be cyclized quantitatively, regardless of the difference in structural constraints imposed on the 7-residue macrocycle by the two different cyanopyridylalanine residues (Figure 2e and f). 


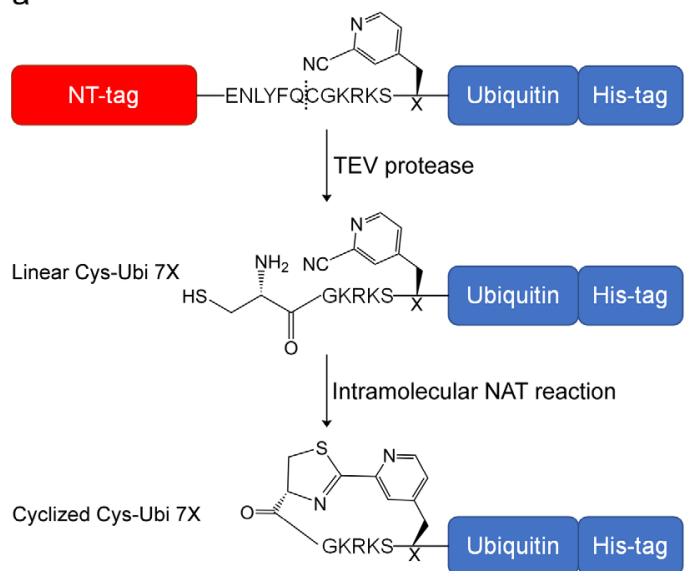

b

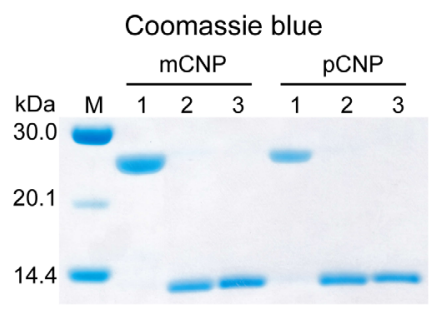

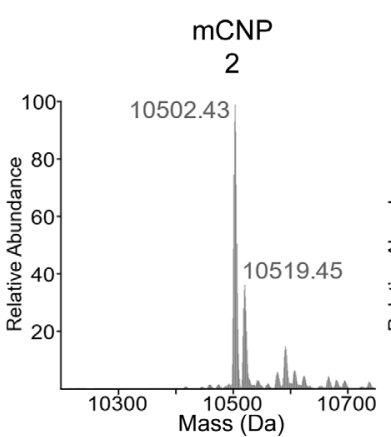

e

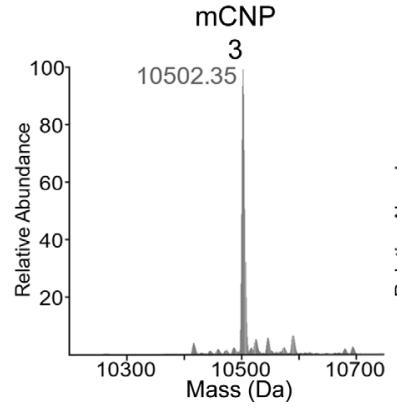

d

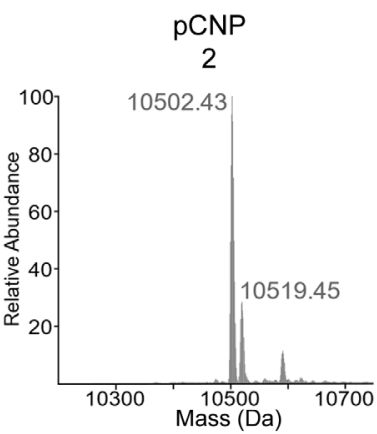

f

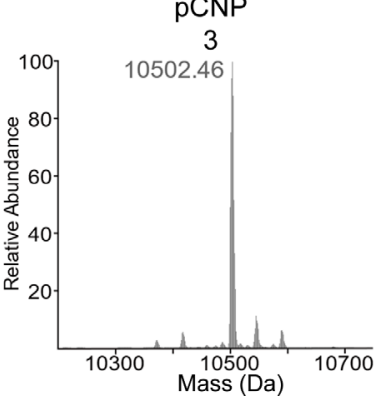

Figure 2. In vivo protein macrocyclization via intramolecular NAT click reaction. a, Design of the NT-Ubi $7 X$ fusion protein used in the current study. X indicates the position of the ncAA. b, SDS-PAGE analysis: M, protein molecular weight marker (the size of each band is indicated on the left); lane 1, NT-Ubi $7 X$ before TEV protease cleavage; lane 2, NT-Ubi $7 \mathrm{X}$ after TEV protease cleavage for $4 \mathrm{~h}$ at $25^{\circ} \mathrm{C}$; lane 3 , purified Cys-Ubi X7 expressed using the 3 -plasmid system for in vivo protein macrocyclization. c-f, Intact protein mass spectra of the Cys-Ubi X7 samples shown in lanes 2 and 3 of b. Expected masses of linear and cyclized Cys-Ubi X7 are 10519.94 and $10502.91 \mathrm{Da}$, respectively.

An alternative way of polypeptide cyclization is achieved by a tandem NAT click reaction between a polypeptide containing two cyanopyridylalanine residues and a bi-functional aminothiol reagent. We used ethylenediamine dicysteine (EDDC), where the carboxyl groups of two cysteine residues are linked by an ethylenediamine moiety (Figure 3a, Supporting Information). The approach takes advantage of the difference in reaction rate between the slower intermolecular and faster intramolecular NAT reaction, where EDDC first reacts with a single cyanopyridylalanine moiety to form a singly tagged protein and this mono-functionalized intermediate undergoes a fast, spontaneous intramolecular NAT reaction with the second cyanopyridylalanine moiety. As a result, formation of the cyclized product is strongly favoured over doubly tagged uncyclized product even in the presence of a large excess of the diaminothiol reagent. The scheme was successful with all three proteins tested, which were produced with two mCNP residues (GB1 A24X/K28X, RFP 237X/243X and RFP A204X/237X, where $X=m C N P)$. Following incubation with $5 \mathrm{mM}$ EDDC (10 $\mathrm{mM}$ for RFP $237 \mathrm{X} / 243 \mathrm{X}$ ) at $25^{\circ} \mathrm{C}$, the reactions were complete after $4 \mathrm{~h}$ in near-quantitative yields as indicated by intact protein mass spectrometry (Figure 3b-d).

The success of our system for genetic encoding of cyanopyridylalanines in high yield is based on (i) our PyIRS library derived from the methanogenic archaeon ISO4-G1, which proved exceptionally adaptable for encoding aromatic ncAAs, and (ii) a two-plasmid selection system established previously for a chimeric PyIRS variant tailored to the genetic encoding of lysinebased ncAAs. ${ }^{[10 b]}$ The versatility of G1PyIRS systems has been demonstrated previously in bacteria, ${ }^{[16]}$ mammalian cells, ${ }^{[17]}$ and cell-free protein synthesis. ${ }^{[10 b]}$

The ncAAs mCNP and pCNP present a balanced compromise between biocompatibility and reactivity. They are non-toxic in vivo and the activated nitrile functionality does not react with functional groups found in cellular biopolymers. In contrast, the activated nitrile functionality ligates readily and in high yield with 1,2-aminothiol compounds, provided they are present in high local concentration, as achieved in, e.g., intramolecular reactions. In $E$. coli, the cyanopyridylalanine ncAAs appear to be resistant against reaction with metabolic compounds, including cysteine present at natural intracellular concentrations. This is an advantage over ncAAs with a azide group, which are susceptible to chemical reduction in bacterial cells, ${ }^{[4 \mathrm{~b}, 18]}$ or trans-cyclooctene (TCO) groups, which have been shown to be prone to isomerization to the non-reactive ciscyclooctene isomer in vivo. ${ }^{[19]}$ Previous attempts to genetically encode the 1,2-aminothiol group as a reactive conjugation group proved unsuccessful due to reaction of the aminothiol moiety with pyruvate, which is abundant in cells. ${ }^{[20]}$ A genetic encoding system subsequently developed for a chemically caged 1,2aminothiol functionality depends on the provision of chemicals for decaging, compromising in vivo applications. ${ }^{[21]}$ 
a
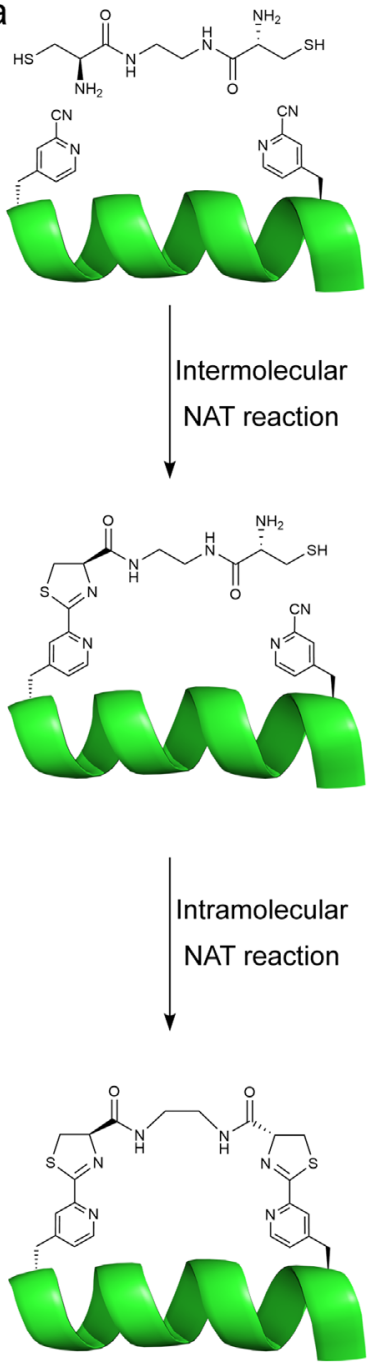

b

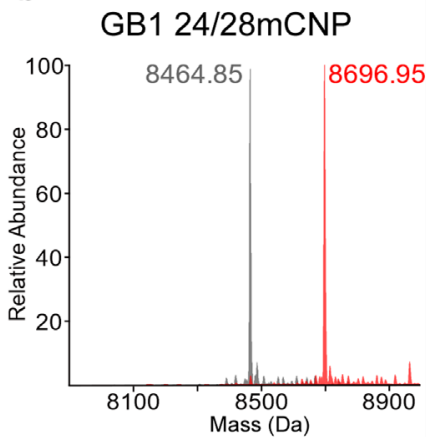

C

RFP 237/243mCNP

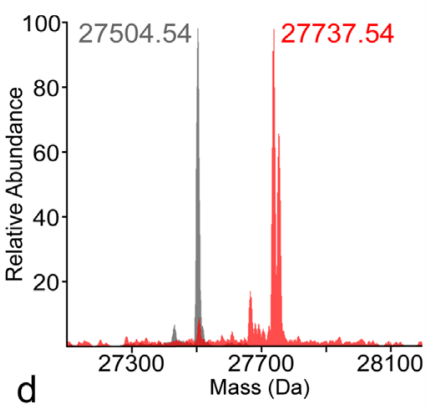

RFP 204/237mCNP

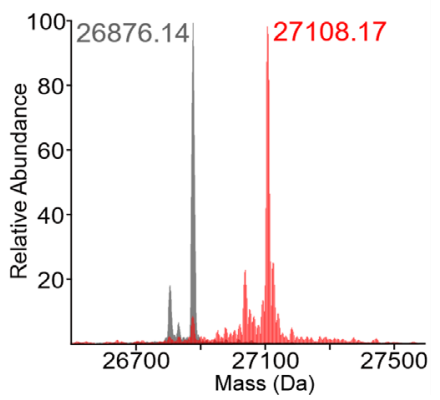

Figure 3. Stapling by tandem intermolecular-intramolecular NAT reaction. a, Intermolecular NAT reaction of EDDC results in the formation of singly tagged protein, which spontaneously undergoes an intramolecular NAT reaction to form the cyclized product. b-d, Intact protein mass spectrum analysis of the reaction between GB1 A24X/K28X, RFP 237X/243X, or RFP A204X/237X after incubation with EDDC for $4 \mathrm{~h}$ at $25{ }^{\circ} \mathrm{C}$. Expected masses of the unreacted proteins and the cyclized products are reported in Table S4.

The present work expands the number of genetically encoded noncanonical amino acids by two different versions of cyanopyridylalanine, which are privileged for NAT click reactions owing to a reactive nitrile group, which spontaneously reacts with 1,2-aminothiol compounds. Incorporation of mCNP or pCNP residues in a protein offers a multitude of possibilities for its sitespecific conjugation with different tags under physiological conditions, which may contain fluorescent or other reporter groups. As the cyanopyridylalanine residues react with $\mathrm{N}$-terminal cysteine, they afford a new approach for peptide and protein cyclisation. Two cyanopyridylalanine residues in a polypeptide chain provide a means for polypeptide stapling.

The NAT click reaction between cyanopyridylalanine residues and 1,2-aminothiols proceeds spontaneously after simple mixing. Unlike the copper catalyst in CuAAC reactions, which tends to generate reactive oxygen species that degrade proteins and are toxic to cells, ${ }^{[22]}$ the absence of added catalyst in the NAT click reaction is a considerable advantage in modifying proteins within living cells. Beyond protein tagging, cyanopyridylalanines enable efficient protein macrocyclization in vitro and in cells, which we envisage to afford a convenient protein engineering tool to enhance the thermal and proteolytic stability of proteins, as well as for the in vivo generation of libraries of macrocyclic peptides and proteins. ${ }^{[23]}$

In conclusion, the biocompatible NAT click reaction between genetically encoded cyanopyridylalanine ncAAs and aminothiols offers an attractive tool for intra- and extra-cellular bioconjugation. The advantages offered by the NAT click reaction over previously reported bioorthogonal reactions makes it a highly valuable alternative to available protein ligation tools.

\section{Acknowledgements}

We thank Dr. Harpreet Vohra and Michael Devoy at the John Curtin School of Medical Research, Australian National University for technical support on FACS experiments. This re-search was undertaken in part using the MX2 beamline at the Australian Synchrotron, part of ANSTO, and made use of the Australian Cancer Research Foundation (ACRF) detector. Financial support by the Australian Research Council for a Laureate Fellowship to G.O. (FL170100019), a DECRA Fellowship to C.N (DE190100015), projects (DP200100348, DP21010088), and through a Centre of Excellence (CE200100012) is gratefully acknowledged.

Keywords: bioorthogonal reaction $\bullet$ cyanopyridylalanine genetic encoding $\cdot$ noncanonical amino acids $\bullet$ nitrile-aminothiol (NAT) click reaction $\cdot$ protein conjugation

a) R. Rossin, P. Renart Verkerk, S. M. van den Bosch, R. C. M. Vulders, I. Verel, J. Lub, M. S. Robillard, Angew. Chem. Int. Ed. Engl. 2010, 49, 3375-3378; b) C. Guo, H. Kim, E. M. Ovadia, C. M. Mourafetis, M. Yang, W. Chen, A. M. Kloxin, Acta Biomater. 2017, 56, 80-90.

[2] N. K. Devaraj, ACS Cent. Sci. 2018, 4, 952-959.

[3] a) K. Lang, L. Davis, J. Torres-Kolbus, C. Chou, A. Deiters, J. W. Chin, Nat. Chem. 2012, 4, 298-304; b) H. S. Jang, S. Jana, R. J. Blizzard, J. C. Meeuwsen, R. A. Mehl, J. Am. Chem. Soc. 2020, 142, 7245-7249.

[4] a) C. T. Loh, K. Ozawa, K. L. Tuck, N. Barlow, T. Huber, G Otting, B. Graham, Bioconjug. Chem. 2013, 24, 260-268; b) C.-T. Loh, B. Graham, E. H. Abdelkader, K. L. Tuck, G. Otting, Chem. Eur. J. 2015, 21, 5084-5092.

[5] a) M. R. Fleissner, E. M. Brustad, T. Kálai, C. Altenbach, D. Cascio, F. B. Peters, K. Hideg, S. Peuker, P. G. Schultz, W. L. Hubbell, Proc. Natl. Acad. Sci. U.S.A. 2009, 106, 21637 21642; b) E. H. Abdelkader, A. Feintuch, X. Yao, L. A. 
Adams, L. Aurelio, B. Graham, D. Goldfarb, G. Otting, Chem. Commun. 2015, 51, 15898-15901.

[6] a) R. Brabham, M. A. Fascione, Chembiochem 2017, 18 1973-1983; b) J. W. Chin, Nature 2017, 550, 53-60.

[7] a) E. M. Sletten, C. R. Bertozzi, Angew. Chem. Int. Ed. Engl. 2009, 48, 6974-6998; b) C. D. Spicer, E. T. Pashuck, M. M. Stevens, Chem. Rev. 2018, 118, 7702-7743; c) M. L. W. J. Smeenk, J. Agramunt, K. M. Bonger, Curr. Opin. Chem. Biol. 2021, 60, 79-88.

[8] a) D. L. Dunkelmann, J. C. W. Willis, A. T. Beattie, J. W. Chin, Nat. Chem. 2020, 12, 535-544; b) W. E. Robertson, L. F. H. Funke, D. de la Torre, J. Fredens, T. S. Elliott, M. Spinck, Y. Christova, D. Cervettini, F. L. Böge, K. C. Liu, S. Buse, S. Maslen, G. P. C. Salmond, J. W. Chin, Science 2021, 372, 1057-1062.

[9] a) C. Nitsche, H. Onagi, J.-P. Quek, G. Otting, D. Luo, T. Huber, Org. Lett. 2019, 21, 4709-4712; b) R. Morewood, C. Nitsche, Chem. Sci. 2021, 12, 669-674; c) N. A. Patil, J.-P. Quek, B. Schroeder, R. Morewood, J. Rademann, D. Luo, C. Nitsche, ACS Med. Chem. Lett. 2021, 12, 732-737.

a) H. Qianzhu, A. P. Welegedara, H. Williamson, A. E. McGrath, M. C. Mahawaththa, N. E. Dixon, G. Otting, T. Huber, J. Am. Chem. Soc. 2020, 142, 17277-17281; b) E. H. Abdelkader, H. Qianzhu, Y. J. Tan, L. A. Adams, T. Huber, G. Otting, J. Am. Chem. Soc. 2021, 143, 1133-1143.

[11] J. M. Kavran, S. Gundllapalli, P. O'Donoghue, M. Englert, D. Söll, T. A. Steitz, Proc. Natl. Acad. Sci. U.S.A. 2007, 104 11268-11273

[12] Y. Sun, H. Jiang, W. Wu, W. Zeng, X. Wu, Org. Lett. 2013, $15,1598-1601$.

[13] N. Kronqvist, M. Sarr, A. Lindqvist, K. Nordling, M. Otikovs, L. Venturi, B. Pioselli, P. Purhonen, M. Landreh, H. Biverstål, Z. Toleikis, L. Sjöberg, C. V. Robinson, N. Pelizzi, H. Jörnvall, H. Hebert, K. Jaudzems, T. Curstedt, A. Rising, J. Johansson, Nat. Commun. 2017, 8, 15504.

[14] M. Békés, K. Okamoto, Sarah B. Crist, Mathew J. Jones, Jessica R. Chapman, Bradley B. Brasher, Francesco D. Melandri, Beatrix M. Ueberheide, E. Lazzerini Denchi, Tony T. Huang, Cell Rep. 2013, 5, 826-838.

[15] R. B. Kapust, J. Tözsér, T. D. Copeland, D. S. Waugh Biochem. Biophys. Res. Commun. 2002, 294, 949-955

[16] J. C. W. Willis, J. W. Chin, Nat. Chem. 2018, 10, 831-837.

[17] B. Meineke, J. Heimgärtner, J. Eirich, M. Landreh, S. J. Elsässer, Cell Rep. 2020, 31, 107811.

[18] K. L. Kiick, E. Saxon, D. A. Tirrell, C. R. Bertozzi, Proc. Natl. Acad. Sci. U.S.A. 2002, 99, 19-24

[19] R. Rossin, S. M. van den Bosch, W. ten Hoeve, M. Carvelli, R. M. Versteegen, J. Lub, M. S. Robillard, Bioconjugate Chem. 2013, 24, 1210-1217.

[20] I. E. Gentle, D. P. De Souza, M. Baca, Bioconjugate Chem. 2004, 15, 658-663. a) D. C. Kennedy, C. S. McKay, M. C. B. Legault, D. C Danielson, J. A. Blake, A. F. Pegoraro, A. Stolow, Z. Mester, J. P. Pezacki, J. Am. Chem. Soc. 2011, 133 17993-18001; b) Craig S. McKay, M. G. Finn, Chem. Biol. 2014, 21, 1075-1101.

[23] A. Purkayastha, T. J. Kang, Biotechnol. Bioprocess. Eng. 2019, 24, 702-712. 


\section{Entry for the Table of Contents}

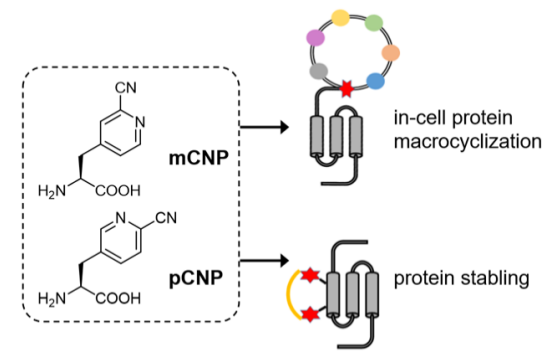

Genetic encoded cyanopyridylalanines are presented as convenient and highly selective reactive groups for in-vivo functionalization, cyclization and stapling of polypeptides. The non-toxic compounds can be genetically incorporated into proteins with high yields and their biocompatible reaction with amino-thiol moieties proceeds under physiological condition to near completion without requiring a catalyst. 


\section{Supporting Information}

\section{Genetic Encoding of Cyanopyridylalanine for In-Cell Protein Macrocyclization by the Nitrile-Aminothiol Click Reaction}

Elwy H. Abdelkader, ${ }^{1 \dagger}$ Haocheng Qianzhu, ${ }^{2 \dagger}$ Josemon George, ${ }^{2}$ Rebecca L. Frkic, ${ }^{1}$

Colin J. Jackson, ${ }^{1}$ Christoph Nitsche, ${ }^{2}$ Gottfried Otting, ${ }^{1}$ and Thomas Huber ${ }^{2} *$

1. ARC Centre of Excellence for Innovations in Peptide \& Protein Science, Research School of Chemistry, Australian National University, Canberra, ACT 2601, Australia

2. Australian National University, Research School of Chemistry, Canberra, ACT 2601, Australia

${ }^{\dagger}$ These authors contributed equally to this work.

*e-mail: t.huber@anu.edu.au 


\section{Table of Contents:}

\section{Supplementary Methods}

Screening of functional G1PylRS mutants that recognize cyanopyridylalanine ncAAs by FACS

Construction of the library plasmid and selection plasmid

Selection of active and specific G1PylRSs for $\mathrm{mCNP}$

Selection of active and specific G1PylRSs for $p C N P$

In vivo protein expression and purification

Protein tagging with aminothiols

Expression, purification, and crystallization of G1mCNPRS

Intact protein mass spectrometry

Figure S1. Sequence alignment of MmPylRS and G1PylRS

Figure S2. Substrate binding pocket of MmPylRS (PDB: 2Q7E)

Figure S3. FACS enrichment during G1mCNPRS selection process

Figure S4. FACS enrichment during G1pCNPRS selection process

Figure S5. Crystal structure of G1mCNPRS (PDB: 7R6O)

Figure S6. Effect of ncAA concentration on the expression yield of RFP 13X

Figure S7. Effect of ncAA concentration on E. coli cell growth

Figure S8. Intact protein mass spectra of the fusion protein NT-Ubi 7X

Table S1. Mutations found in the 7 selected G1PylRSs that recognize mCNP

Table S2. Mutations found in the 17 selected G1PylRSs that recognize pCNP

Table S3. Yields of the proteins expressed in the present study

Table S4. Expected mass of the proteins reported in the present study

Table S5. Complete nucleotide sequences of the plasmids

Table S6. DNA and corresponding amino acid sequences of the proteins

Table S7. List of primers used in the present study

Table S8. Structural data of the single-crystal X-ray structure of G1mCNPRS

Synthesis of EDDC (ethylenediamine dicysteine)

Synthesis of mCNP (L-3-(2-cyano-4-pyridyl)alanine)

\section{References}




\section{Supplementary Methods}

\section{Screening of functional G1PylRS mutants that recognize cyanopyridylalanine ncAAs by FACS}

\section{Construction of the library plasmid and selection plasmid}

A pBK-G1RS plasmid was first constructed by introducing a wild-type G1PylRS gene (G1RSwt) under a mutant $G \ln S$ promoter $\left(G \ln S^{\prime}\right)$ into a $\mathrm{pBK}$ vector. ${ }^{1-3}$ An expression cassette for ${ }^{\mathrm{G} 1 \mathrm{Pyl}}{ }^{\mathrm{tRNA}} \mathrm{CUA}$ under the control of the lpp promoter was cloned into the vector in the opposite direction of the synthase gene. From the structure of the G1PylRS's homologues MmPylRS, seven residues were identified to directly interact with the bound amino acid substrate (Figure S1 and Figure S2). The equivalent set of residues in G1PylRS are L124, Y125, N165, V167, Y204, A221, and W237. Randomized triplet NNK codons $(\mathrm{N}=\mathrm{A}+\mathrm{G}+\mathrm{T}+\mathrm{C}, \mathrm{K}=\mathrm{G}+\mathrm{T})$ were introduced for full randomization at four mutation sites: L124, Y125, A221, and W237, using doped oligonucleotide primers containing these codons. To avoid most of the bulky or charged amino acids at position N165 and V167, which are located in the $\beta$-strand closest to the bound substrate, N165 was selectively mutated to $\mathrm{G} / \mathrm{A} / \mathrm{V} / \mathrm{S} / \mathrm{N} / \mathrm{T} / \mathrm{I} / \mathrm{D}$ through randomized triplet codons $\mathrm{RNC}$ (R $=\mathrm{A}+\mathrm{G}) ; \mathrm{V} 167$ was similarly mutated to $\mathrm{G} / \mathrm{A} / \mathrm{V} / \mathrm{S} / \mathrm{C} / \mathrm{L} / \mathrm{F}$ through codon $\mathrm{KBM}(\mathrm{B}=\mathrm{C}+$ $\mathrm{G}+\mathrm{T}, \mathrm{M}=\mathrm{A}+\mathrm{C}$ ). To further reduce the size of the library, residue Y204, which is equivalent to Y384 in MmPylRS, was selectively mutated to aromatic residues (F/Y/W), which we hypothesized to have favorable interactions with the aromatic ring of the substrate via $\pi$-stacking. The selective mutation from Y204 to F/Y/W was conducted by mixing three different gene fragments containing either Y204F, Y204Y, or Y204W as one of the PCR templates when conducting overlap PCR for the whole fragment assembly of 
the G1PylRS library. This library theoretically encodes $2.688 \times 10^{7}$ variants of G1PylRS mutants.

The G1PylRS library fragment was ligated back into a pBK vector in the same way as described in our previous work..$^{2-3}$ The actual resulting library contained approximately $10^{9}$ individual transformants. DNA sequencing of randomly picked colonies revealed no significant bias. All primers and gene fragments were commercially synthesized (Integrated DNA Technologies, USA).

A pBAD-H6RFP plasmid encoding a His6-amber(TAG)-mCherry red fluorescent protein (H6RFP) reporter gene under the control of the $\operatorname{araBAD}$ promoter was used as the selection plasmid. ${ }^{3}$

\section{Selection of active and specific G1PylRSs for $\mathrm{mCNP}$}

To carry out the selection, the library plasmid pBK-G1RS was transformed into $E$. coli DH10B cells harboring the selection plasmid pBAD-H6RFP. Following recovery from transformation, the culture was directly inoculated into a flask with $25 \mathrm{~mL}$ LB medium containing $100 \mathrm{mg} / \mathrm{L}$ carbenicillin and $50 \mathrm{mg} / \mathrm{L}$ kanamycin, supplied with $0.4 \%$ L-arabinose and $3 \mathrm{mM} \mathrm{mCNP}$, which served as the sample for the first round of positive selection (1P+). Overnight expression at $37{ }^{\circ} \mathrm{C}$ led to a well-detectable level of RFP expression. Cells were resuspended in $7.5 \mathrm{~mL}$ PBS buffer $(137 \mathrm{mM} \mathrm{NaCl}, 2.7 \mathrm{mM} \mathrm{KCl}$, $10 \mathrm{mM} \mathrm{Na}_{2} \mathrm{HPO}_{4}, 1.8 \mathrm{mM} \mathrm{KH}_{2} \mathrm{PO}_{4}, \mathrm{pH}$ 7.4) after harvesting. A 100-fold dilution yielded a concentration suitable for cell sorting by FACS (Figure S3) on an Aria II high speed cell sorter (BD Biosciences, USA).

The top $1.1 \%$ of cells with high RFP levels were selected from $\mathbf{1 P +}$, collecting $8.0 \times 10^{5}$ cells in $90 \mathrm{~min}$. The cells collected were subjected to the following round of negative selection without the addition of $\mathrm{mCNP}$ and regrew as sample $\mathbf{2} \mathbf{N}-$, from where 
cells with low RFP expression levels $\left(69.4 \%\right.$ of the total) were collected $\left(2.0 \times 10^{6}\right.$ cells in $12 \mathrm{~min})$. These cells were aliquoted to inoculate media with positive $(\mathbf{3 P}+)$ and negative (3P-) conditions. The top $13.5 \%$ of RFP-positive cells from $\mathbf{3 P}+$ were collected $\left(1.0 \times 10^{6}\right.$ cells in $20 \mathrm{~min}$ ) and recovered under negative condition to get $\mathbf{4 N}-$. Following sorting $\left(2.0 \times 10^{6}\right.$ cells in $\left.10 \mathrm{~min}\right), 78.0 \%$ of the cells showing the lowest level of RFP fluorescence were selected from $\mathbf{4 N}$-. They were aliquoted to be recovered as $\mathbf{5 P +}$ and $\mathbf{5 P}-$, respectively. At this stage, the number of RFP fluorescent cells was more than thirty-fold higher in the $\mathbf{5 P +}$ than in the $\mathbf{5 P}$ - sample (33.5\% compared to $0.9 \%)$. $1.0 \times 10^{5}$ cells collected in 10 minutes from the top 5.0\% RFP fluorescent cells of the $\mathbf{5 P}+$ sample were recovered for storage. An aliquot of 5,000 cells were allowed to recover on LB agar plates containing $100 \mathrm{mg} / \mathrm{L}$ carbenicillin and $50 \mathrm{mg} / \mathrm{L}$ kanamycin, and individual clones were analyzed using 96 -well plates. 120 candidates were inoculated into both positive $(+3 \mathrm{mM}$ $\mathrm{mCNP}$ ) and negative (without mCNP) growth conditions. The fluorescence level was measured after expression overnight, using a TECAN Infinite 200 Pro M Plex plate reader (Tecan, Switzerland) and normalized by the $\mathrm{OD}_{600}$ of the cell culture. 7 candidates with the highest RFP level in the positive condition were chosen for sequencing. 6 individually different sequences were found (Table S1). One mutation set was identified twice as G1mCNP16 and G1mCNP34 (L124A, Y125L, V167A, Y204W, A221S). G1mCNP34 was used in the subsequent experiments as the best functional G1mCNPRS.

\section{Selection of active and specific G1PylRSs for pCNP}

The preparation method for the $\mathbf{1 P +}$ sample of selection for functional pCNPRS was the same as for mCNPRS, except for providing 3mM pCNP (Chem-Space, Latvia (XV6117)) instead of mCNP during overnight expression. 
The top $0.3 \%$ of cells with high RFP levels were selected from the $\mathbf{1 P +}$ round, collecting $2.0 \times 10^{5}$ cells in 2 hours (Figure $\mathbf{S 4}$ ). The cells collected were subjected to the following round of negative selection without the addition of $\mathrm{pCNP}(\mathbf{2 N}-)$, from where cells with low RFP expression levels $\left(85.6 \%\right.$ of the total) were collected $\left(2.0 \times 10^{6}\right.$ cells in $8 \mathrm{~min})$. These cells were aliquoted to inoculate media with positive (3P+) and negative (3P-) conditions. The top $0.9 \%$ of RFP-positive cells from the $\mathbf{3 P}+$ round were collected $\left(2.0 \times 10^{5}\right.$ cells in 1 hour) and recovered under negative condition to generate $4 \mathrm{~N}$-. Following sorting $\left(2.0 \times 10^{6}\right.$ cells in $\left.8 \mathrm{~min}\right), 85.6 \%$ of the cells showing the lowest level of RFP fluorescence were selected. They were aliquoted to be recovered for the samples $\mathbf{5 P +}$ and 5P-. At this stage, the difference in RFP expression level was clear (40.1\% compared to $30.6 \%$ ). The selection process was ended after this round, as sequencing suggested that the remaining sequence diversity encompassed fewer than ten different mutants. $1.0 \times 10^{5}$ cells collected in 15 min from the top 5.0\% RFP fluorescent cells of the $\mathbf{5 P}+$ sample were recovered for storage. An aliquot of 5,000 cells were allowed to recover on LB agar plates containing $100 \mathrm{mg} / \mathrm{L}$ carbenicillin and $50 \mathrm{mg} / \mathrm{L}$ kanamycin, and individual clones were analyzed using 96-well plates. 120 candidates were inoculated into both positive $(+3 \mathrm{mM}$ pCNP) and negative (without pCNP) growth conditions. The fluorescence level was measured after expression overnight, using a TECAN Infinite 200 Pro M Plex plate reader (Tecan, Switzerland) and normalized by the $\mathrm{OD}_{600}$ of the cell culture. 17 candidates with the highest RFP level in the positive condition were chosen for sequencing (Table S2). 3 individually different mutation sets were found: (1) L124A, Y125F, Y204W, W237Y; (2) L124A, Y125F, Y204W, A221S, W237Y; (3) L124S, Y125F, Y204W, A221S, W237Y. G1pCNP37 was used for following experiment as the best functional G1pCNPRS. 


\section{In vivo protein expression and purification}

For site-specific incorporation of $\mathrm{mCNP}$ and $\mathrm{pCNP}$, E. coli $\mathrm{B}-95 . \Delta \mathrm{A}$ cells ${ }^{4}$ were co-transformed with pRSF-G1mCNPRS or pRSF-G1pCNPRS, respectively, and the pCDF plasmid containing the amber codon-interrupted gene of the protein of interest. The cells were grown at $37{ }^{\circ} \mathrm{C}$ in $\mathrm{LB}$ medium containing $25 \mathrm{mg} / \mathrm{L}$ kanamycin and $25 \mathrm{mg} / \mathrm{L}$ spectinomycin. An aliquot $(0.5 \mathrm{~mL})$ of an overnight culture was used to inoculate $50 \mathrm{~mL}$ LB medium supplemented with $25 \mathrm{mg} / \mathrm{L}$ kanamycin, $25 \mathrm{mg} / \mathrm{L}$ spectinomycin, and $1 \mathrm{mM}$ $\mathrm{mCNP}$ or $2 \mathrm{mM}$ pCNP. The cells were grown at $37{ }^{\circ} \mathrm{C}$ to an $\mathrm{OD}_{600}$ of $0.6-1$. At this point, the temperature was reduced to $25^{\circ} \mathrm{C}$ and protein expression was induced by the addition of $1 \mathrm{mM}$ isopropyl $\beta$-D-1-thiogalactopyranoside (IPTG).

To produce the wild-type proteins, the respective genes were cloned between the NdeI and BamHI restriction sites of the pET-3a expression plasmid (Novagen, USA). The corresponding pET-3a plasmid was then transformed into E. coli BL21(DE3) cells and the cells were recovered at $37{ }^{\circ} \mathrm{C}$ in $\mathrm{LB}$ medium containing $100 \mathrm{mg} / \mathrm{L}$ carbenicillin. An aliquot $(0.5 \mathrm{~mL})$ of an overnight culture was used to inoculate $50 \mathrm{~mL}$ LB medium supplemented with $100 \mathrm{mg} / \mathrm{L}$ carbenicillin. The cells were grown at $37^{\circ} \mathrm{C}$ to an $\mathrm{OD}_{600}$ of 0.6-1. Afterwards, the temperature was reduced to $25^{\circ} \mathrm{C}$ and protein expression was induced by the addition of $1 \mathrm{mM}$ IPTG.

After protein expression for $16 \mathrm{~h}$, the cells were harvested by centrifugation at $4000 \mathrm{~g}$ for 15 minutes at $4{ }^{\circ} \mathrm{C}$. Following resuspension in buffer A $(50 \mathrm{mM}$ Tris- $\mathrm{HCl} \mathrm{pH}$ 7.5, $300 \mathrm{mM} \mathrm{NaCl}, 5 \%$ glycerol, $10 \mathrm{mM}$ imidazole), the cells were lysed using an Avestin Emulsiflex C5 (Avestin, Canada) using two passes at 10,000-15,000 psi. The cell lysates were centrifuged for $1 \mathrm{~h}$ at $30,000 \mathrm{~g}$ at $4{ }^{\circ} \mathrm{C}$. The supernatant was loaded onto a $1 \mathrm{~mL}$ His GraviTrap column (Cytiva, USA). The column was washed with 20 column volumes 
buffer B (same as buffer A but with $20 \mathrm{mM}$ imidazole) and the protein was eluted with 5 column volumes buffer $\mathrm{C}$ (same as buffer A but with $500 \mathrm{mM}$ imidazole). Afterwards, the buffer was exchanged to PBS (137 mM NaCl, $2.7 \mathrm{mM} \mathrm{KCl}, 10 \mathrm{mM} \mathrm{Na}_{2} \mathrm{HPO}_{4}, 1.8 \mathrm{mM}$ $\mathrm{KH}_{2} \mathrm{PO}_{4}, \mathrm{pH}$ 7.4) using an Amicon ultrafiltration centrifugal tube (Merck Millipore, USA) with the appropriate molecular weight cut-off.

\section{Protein tagging with aminothiols}

To a $50 \mu \mathrm{M}$ protein sample in PBS buffer containing $10 \mathrm{mM}$ TCEP, $5 \mathrm{mM}$ cysteine or EDDC was added (from a $100 \mathrm{mM}$ stock solution in PBS). The reaction was incubated with shaking at $25{ }^{\circ} \mathrm{C}$ for $4 \mathrm{~h}$. Next, the buffer was exchanged to PBS using an Amicon centrifugal ultrafiltration tube.

\section{Expression, purification, and crystallization of G1mCNPRS}

For the expression of G1mCNPRS, high cell-density fermentation was used based on the protocol published by Gossert et al. ${ }^{5}$ The gene encoding G1mCNPRS with an Nterminal His 6 tag and TEV cleavage site was cloned between the NdeI and BamHI restriction sites of pET-3a plasmid (Novagen, USA). pET-G1mCNPRS plasmid was transformed into E. coli BL21(DE3) cells. The cells were grown at $37^{\circ} \mathrm{C}$ in LB medium containing $100 \mathrm{mg} / \mathrm{L}$ carbenicillin. Next, the cells were used to inoculate $50 \mathrm{~mL} \mathrm{~TB}$ medium supplemented with $100 \mathrm{mg} / \mathrm{L}$ carbenicillin (pre-culture). Following growth at 37 ${ }^{\circ} \mathrm{C}$ for $16 \mathrm{~h}$, the pre-culture was used to inoculate $950 \mathrm{~mL}$ rich fermenter medium containing $100 \mathrm{mg} / \mathrm{L}$ carbenicillin in a Labfors 5 bioreactor (Infors-HT, Switzerland). The cells were grown at $37{ }^{\circ} \mathrm{C}$ with air flow $=2.5 \mathrm{~L} / \mathrm{min}$, minimal $\mathrm{pO}_{2}=20 \%$, stirring cascade: 500-1200 rpm, and $\mathrm{pH}=7.0$. When the $\mathrm{OD}_{600}$ value of the culture reached 15 , temperature was reduced to $18{ }^{\circ} \mathrm{C}$ and $1 \mathrm{mM}$ IPTG was added for the induction of protein expression. 
After $16 \mathrm{~h}$, the fermentation was stopped, and the cells were harvested by centrifugation at $4000 \mathrm{~g}$ for 15 minutes.

The harvested cells were resuspended in buffer A (50 mM Tris- $\mathrm{HCl} \mathrm{pH} 7.5,300$ $\mathrm{mM} \mathrm{NaCl}, 5 \%$ glycerol, $10 \mathrm{mM}$ imidazole) followed by lysis using an Avestin Emulsiflex C5 (Avestin, Canada; two passes using 10,000-15,000 psi). The clarified cell lysate was loaded onto a 5 mL HisTrap FF column connected to an ÄKTA pure 25 chromatography system (Cytiva, USA). The column was washed with 20 column volumes buffer B (same as buffer A but with $20 \mathrm{mM}$ imidazole) and the protein was eluted with 5 column volumes buffer $\mathrm{C}$ (same as buffer A but with $500 \mathrm{mM}$ imidazole). The eluted protein was desalted using a HiPrep Desalting 26/10 column (Cytiva, USA) equilibrated with buffer D (50 mM Tris- $\mathrm{HCl} \mathrm{pH}$ 7.5, $300 \mathrm{mM}$ sodium chloride, $1 \mathrm{mM}$ dithiothreitol (DTT)) and TEV proteolytic cleavage was done by incubation with TEV protease at $4{ }^{\circ} \mathrm{C}$ for $16 \mathrm{~h}$. The cleaved G1mCNPRS was recovered by a reverse IMAC step.

Finally, G1mCNPRS was loaded onto a HiLoad 26/600 Superdex 200 pg column (Cytiva, USA) equilibrated with buffer E (50 mM Tris- $\mathrm{HCl} \mathrm{pH} 7.5,400 \mathrm{mM}$ sodium chloride). After gel filtration, the fractions containing G1mCNPRS were combined and concentrated to $10 \mathrm{mg} / \mathrm{mL}$ using an Amicon ultrafiltration centrifugal tube (Merck Millipore, USA) with a molecular weight cut-off of $10 \mathrm{kDa}$ and buffer-exchanged to buffer F (50 mM Tris- $\mathrm{HCl}$ pH 7.5, 100 mM NaCl, 3 mM mCNP).

High throughput screening of crystallization conditions was performed using sparse matrix screens Index and Crystal Screen HT (Hampton Research) and SG1 and JCSG (Molecular Dimensions), at $18{ }^{\circ} \mathrm{C}$ with 1:1 ratio of protein:reservoir in a total volume of $1 \mu \mathrm{L}$, using a protein concentration of $10 \mathrm{mg} / \mathrm{ml}$. Crystallization of G1mCNPRS was optimized by hanging-drop vapor diffusion at $18{ }^{\circ} \mathrm{C}$. Large rod-shaped 
orthorhombic crystals were obtained by equilibrating drops of $1 \mu \mathrm{L}$ protein and $1 \mu \mathrm{L}$ reservoir against a solution of 30\% w/v PEG 4000, $100 \mathrm{mM}$ Tris-HCl (pH 9.0), and 200 mM lithium sulfate. Crystals were cryoprotected using Parabar (Hampton Research) prior to flash-freezing in liquid nitrogen.

Diffraction data were collected at $100 \mathrm{~K}$ at the MX2 beamline at the Australian Synchrotron. ${ }^{6}$ Reflections collected were indexed and integrated using $\operatorname{XDS}^{7}$ and scaled in Aimless (CCP4). ${ }^{8}$ The phase problem was overcome by molecular replacement in Phaser MR (CCP4), ${ }^{9}$ using PDB ID 6JP2 with sequence modified in Sculptor ${ }^{10}$ as the search model. The structure was refined by iterative rounds of rebuilding in Coot, ${ }^{11}$ and refinement in phenix.refine. ${ }^{12-13}$ Data collection and refinement statistics are given in Table S8. The completed structure was deposited in the Protein Data Bank (PDB: 7R6O).

\section{Intact protein mass spectrometry}

Intact protein analysis was performed on an Orbitrap Fusion ${ }^{\mathrm{TM}}$ Tribrid $^{\mathrm{TM}}$ mass spectrometer (Thermo Fisher Scientific, USA) connected to a Thermo Fisher Scientific UltiMate 3000 HPLC system equipped with ZORBAX 300SB-C3, $3.5 \mu \mathrm{m}, 4.6$ x 50 mm HPLC column (Agilent Technologies, USA). Approximately 50 pmol of sample was injected using a $500 \mu \mathrm{L} / \mathrm{min}$ linear gradient of solvent $\mathrm{A}(0.1 \%(\mathrm{v} / \mathrm{v})$ formic acid in water) and solvent B $(0.1 \%(\mathrm{v} / \mathrm{v})$ formic acid in acetonitrile), ramping solvent $\mathrm{B}$ from $5 \%$ at the start to $80 \%$ after 12 min. Data were collected using an electrospray ionization (ESI) source in positive ion mode. Protein intact mass was determined by deconvolution using the program Xcalibur 3.0.63 (Thermo Fisher Scientific, USA). 


\section{Supplementary Figures}

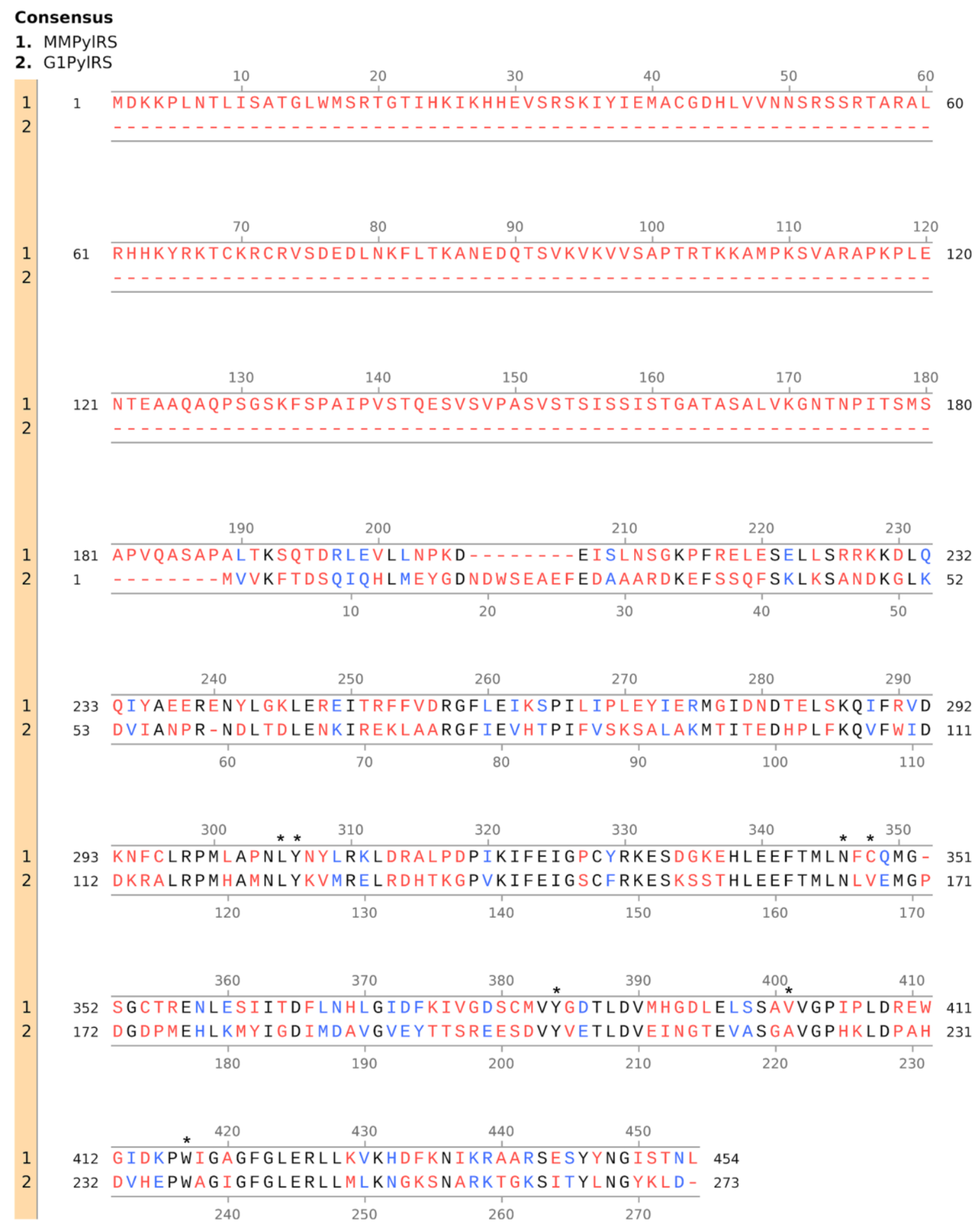

Figure S1. Sequence alignment of MmPylRS and G1PylRS. The alignment was done using the semi-global alignment tool in SnapGene ${ }^{\circledR}$ software (from Insightful Science; available at snapgene.com). Residues referenced in the main text are indicated by asterisks. 


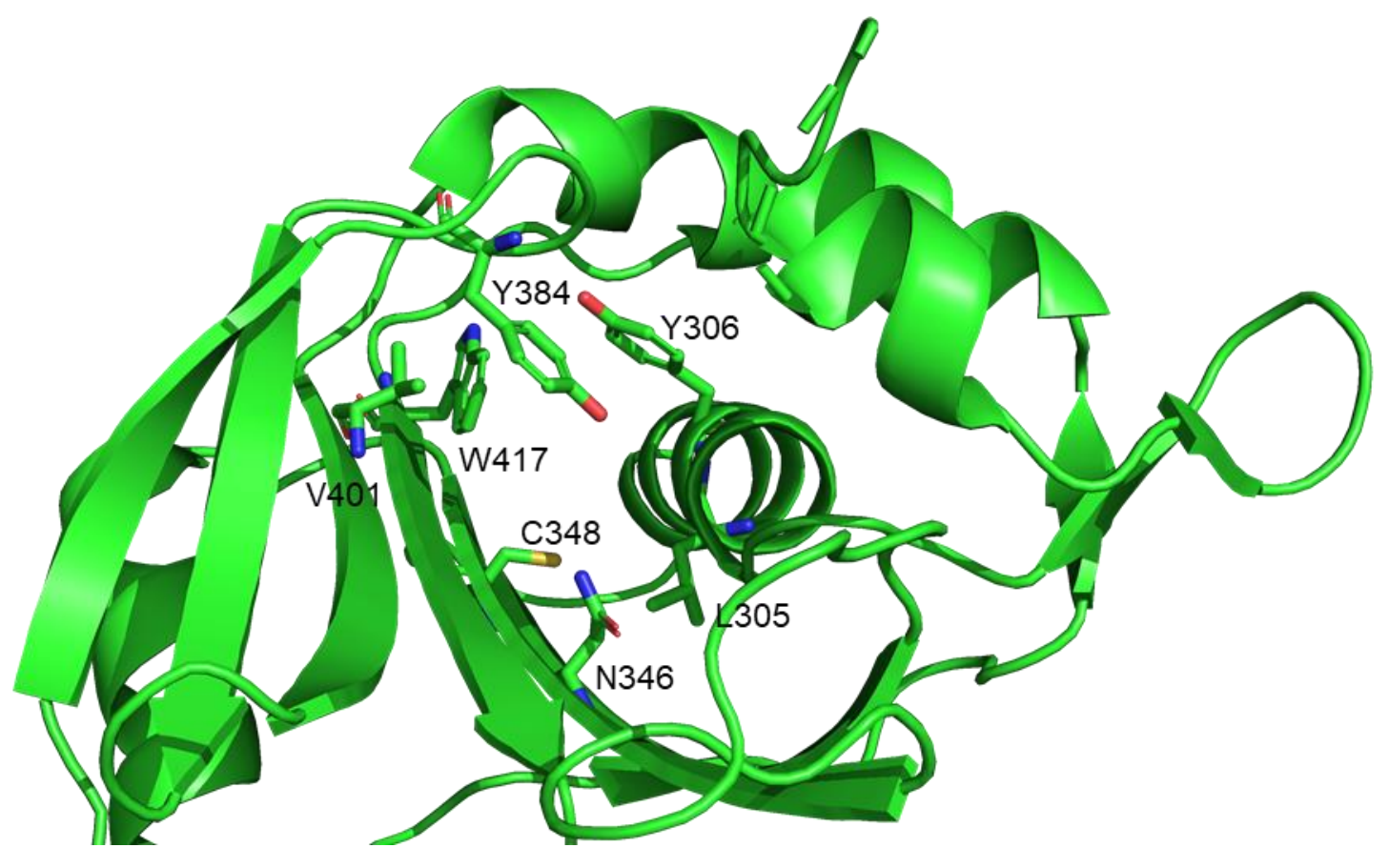

Figure S2. Substrate binding pocket of MmPylRS (PDB: 2Q7E). The residues identified as essential for substrate recognition are shown as sticks. 

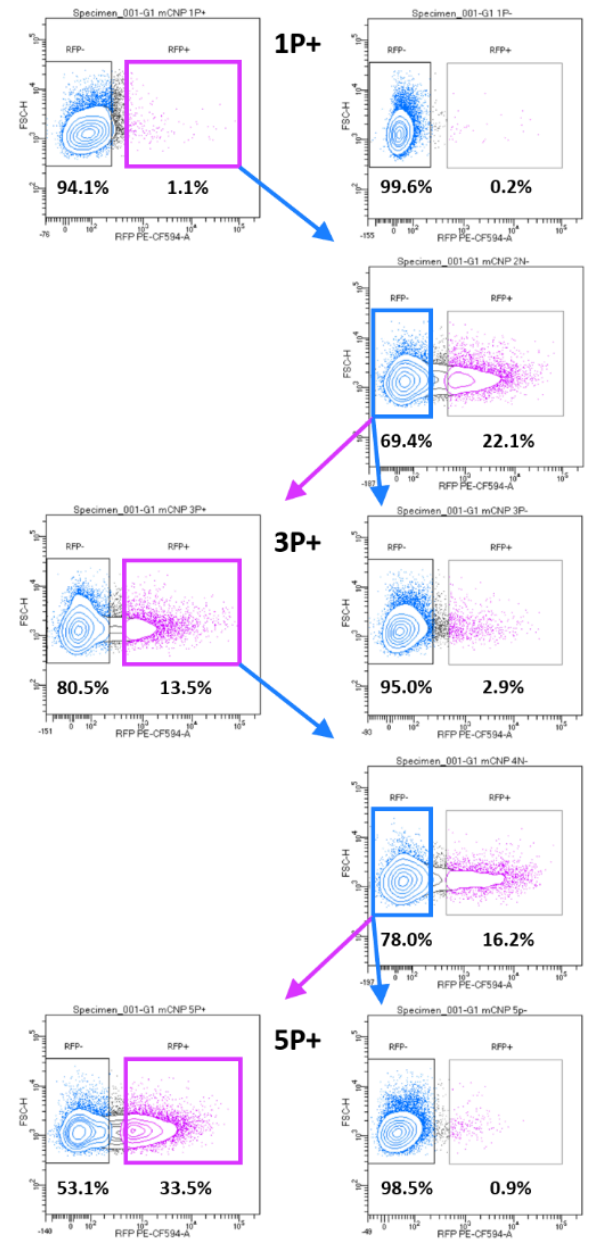

$1 \mathrm{P}-$

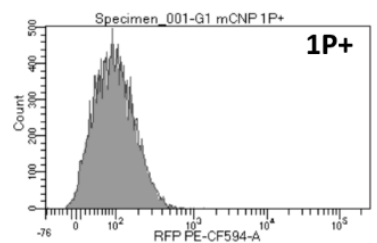

$2 \mathrm{~N}-$

-
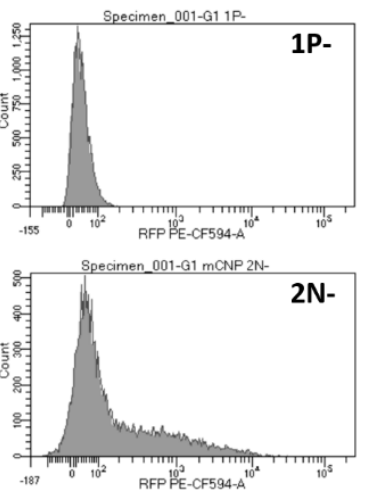

3P-
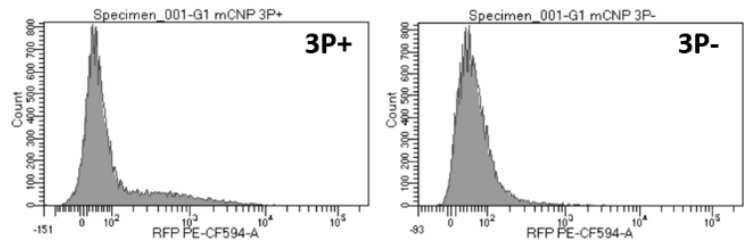

$4 \mathrm{~N}-$

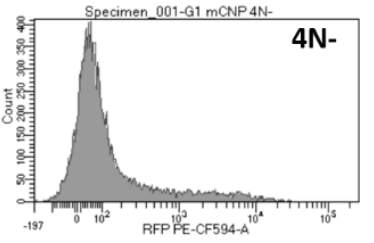

5P.
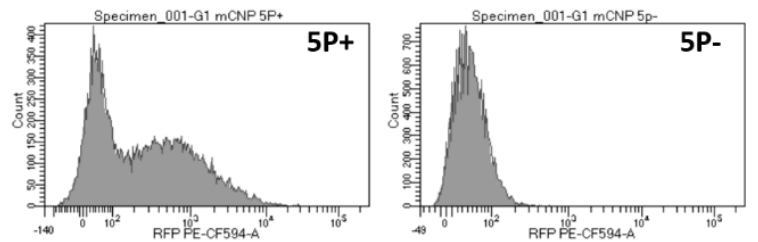

Figure S3. a, FACS enrichment of E. coli cells with active and specific G1PylRS enzymes that recognize mCNP. $\mathbf{P}$ and $\mathbf{N}$ labels refer to positive and negative selection rounds, respectively. Violet/blue frames identify the fractions collected from each positive/negative round. +/- labels and violet/blue arrows refer to cell growth in the presence or absence of target ncAA (herein, $3 \mathrm{mM}$ of $\mathrm{mCNP}$ ). The vertical axis plots the forward scatter that reports on cell size and the horizontal axis indicates the level of RFP fluorescence. $33.5 \%$ of the $\mathbf{5 P}+$ sample with the brightest RFP fluorescence level were collected and further characterized. b, Histograms of cell counts in all five rounds of mCNPRS selection. At the fifth round of positive selection, the ratio of cells with high RFP fluorescence was much greater in the presence of mCNP $(\mathbf{5 P +})$ than in the absence of $\mathrm{mCNP}$ (5P-). 
$\mathbf{a}$
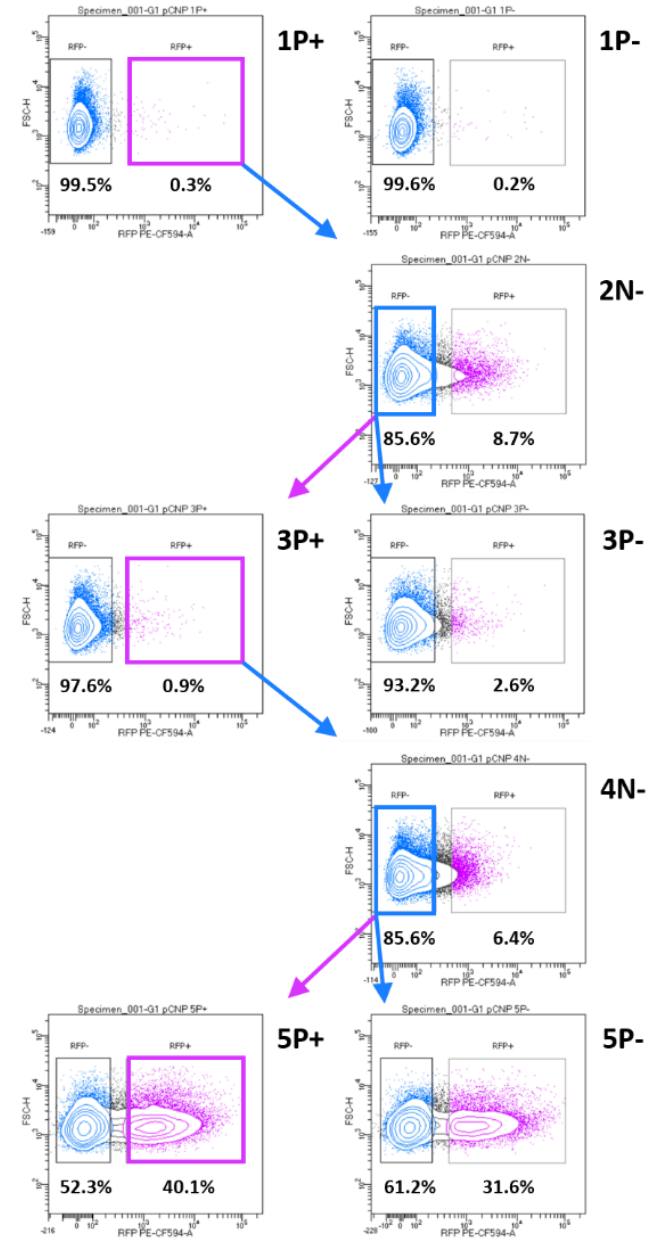
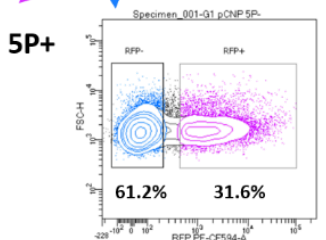

1P-

$2 \mathrm{~N}-$

3P.

$4 \mathrm{~N}-$

$5 P$ - b
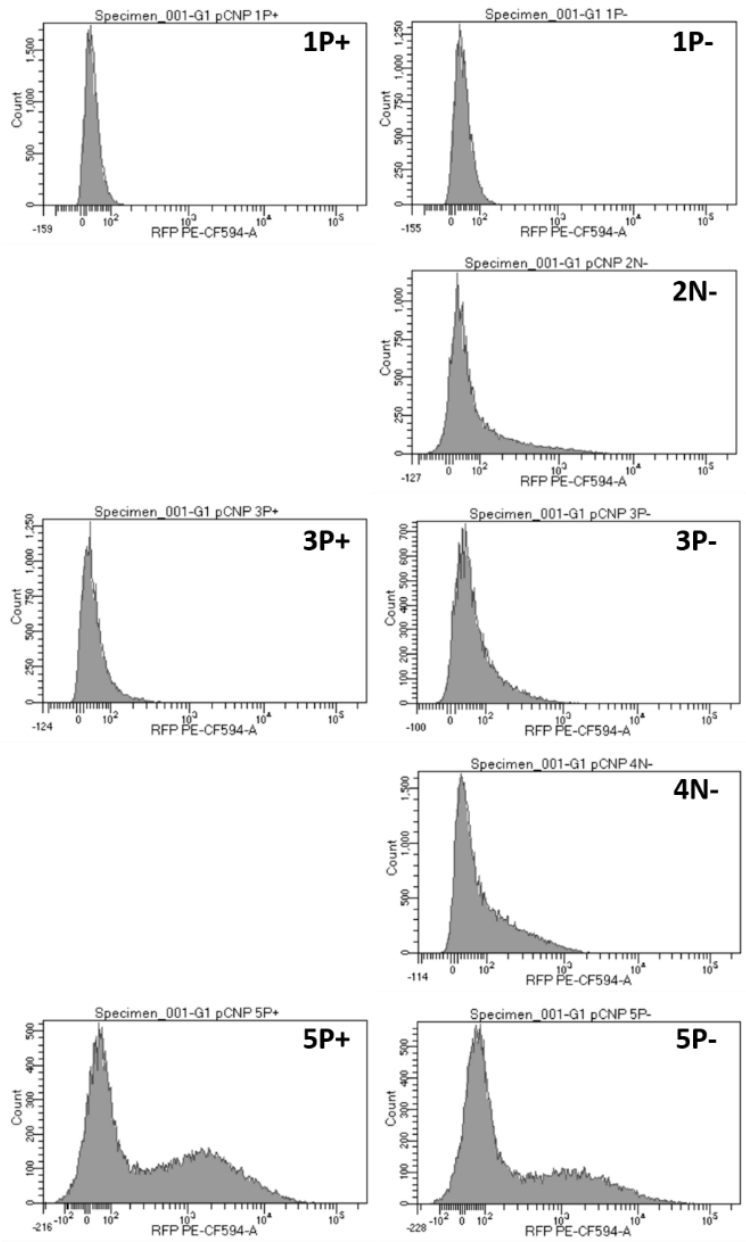

Figure S4. a, FACS enrichment of E. coli cells with G1PylRS enzymes specific for pCNP. $\mathbf{P} / \mathbf{N}$ and $+/$ - labels, violet/blue frames and arrows have the same indication as in Figure S3. b, Histograms of cell counts in all five rounds of selection for active and specific G1pCNPRS. The ratio of cells with high RFP fluorescence in the $\mathbf{5 P}+$ sample $(40.1 \%)$ was 1.27 times higher than in the 5P-sample (31.6\%), which indicates a good chance of selecting true-positive candidates during further characterization. 

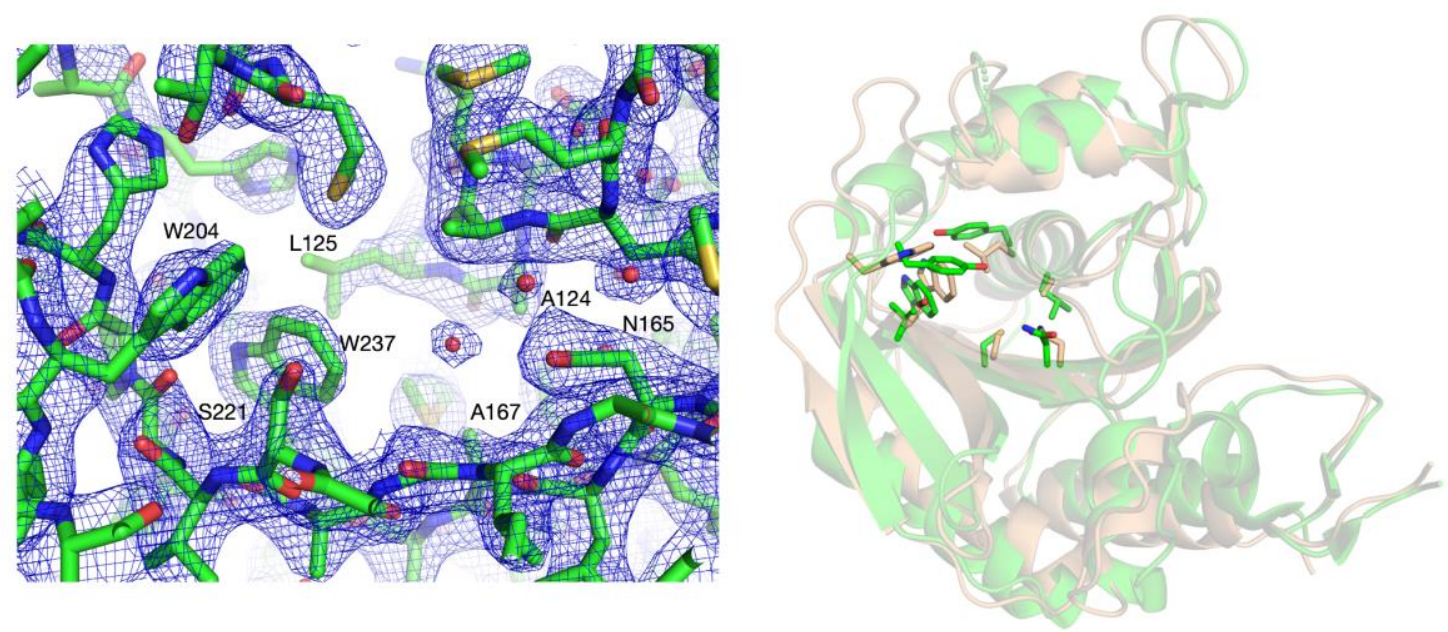

Figure S5. The crystal structure of G1mCNPRS (PDB: 7R6O). a, The amino acid binding site of G1mCNPRS, with $2 m \mathrm{~F}_{\mathrm{o}}-D \mathrm{~F}_{\mathrm{c}}$ electron density, showing the binding site is well resolved in the crystal structure but no significant electron density for the m-CNP substrate is observed. Residues that were mutated (A124, L125, N165, A167, W204, W237, A167) are labelled. $b$, The structure of the amino acid binding domain of the G1mCNP tRNA synthetase (beige) is highly conserved, and superimposes with $1.63 \AA$ C $\alpha$-RMSD over 186 residues on the C-terminal domain of the pyrrolysyl-tRNA synthetase from M. mazei (PDB: 2Q7E; green). ${ }^{14}$ Residues that were mutated (A124, L125, N165, A167, W204, W237, A167) are shown as sticks. 
a

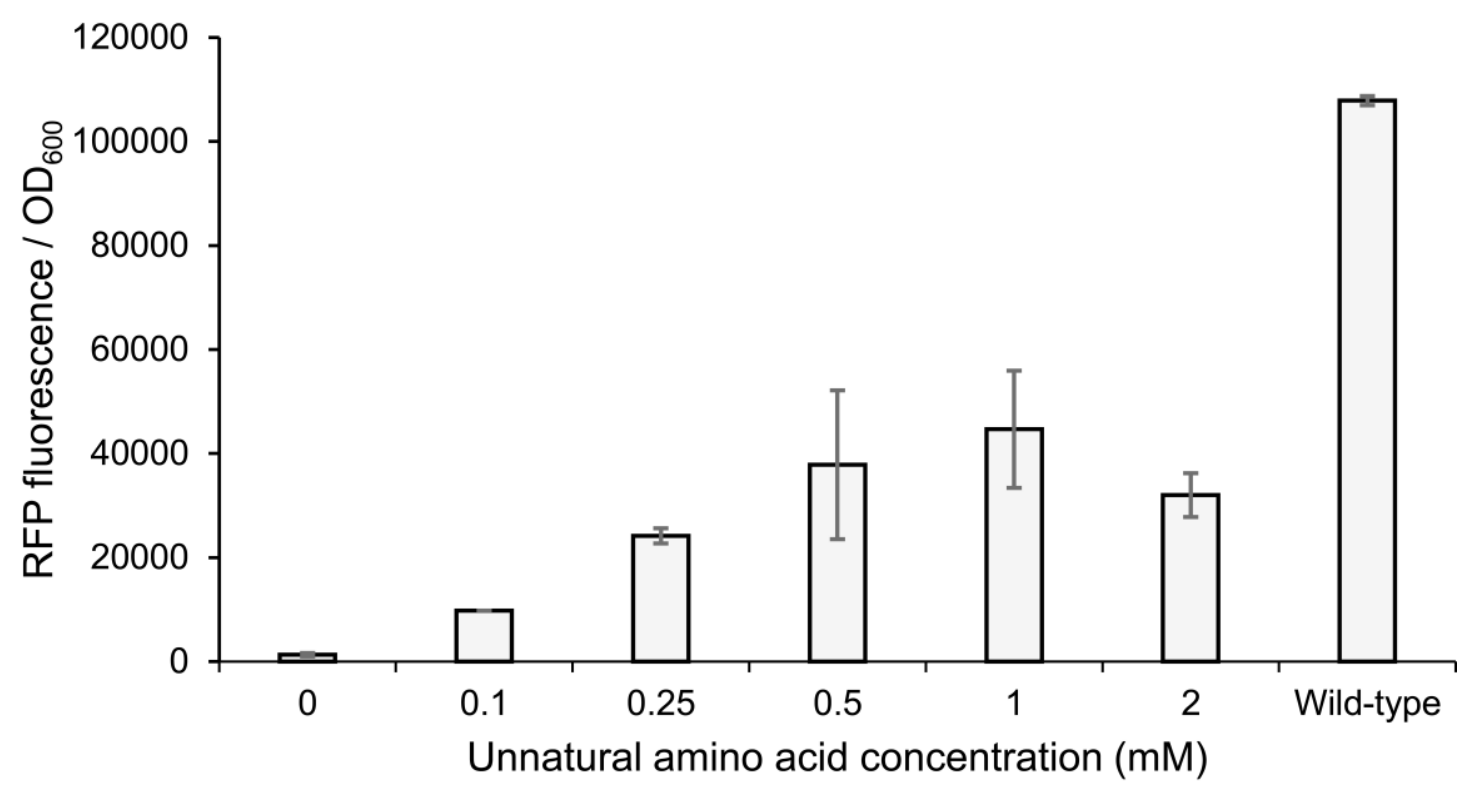

b

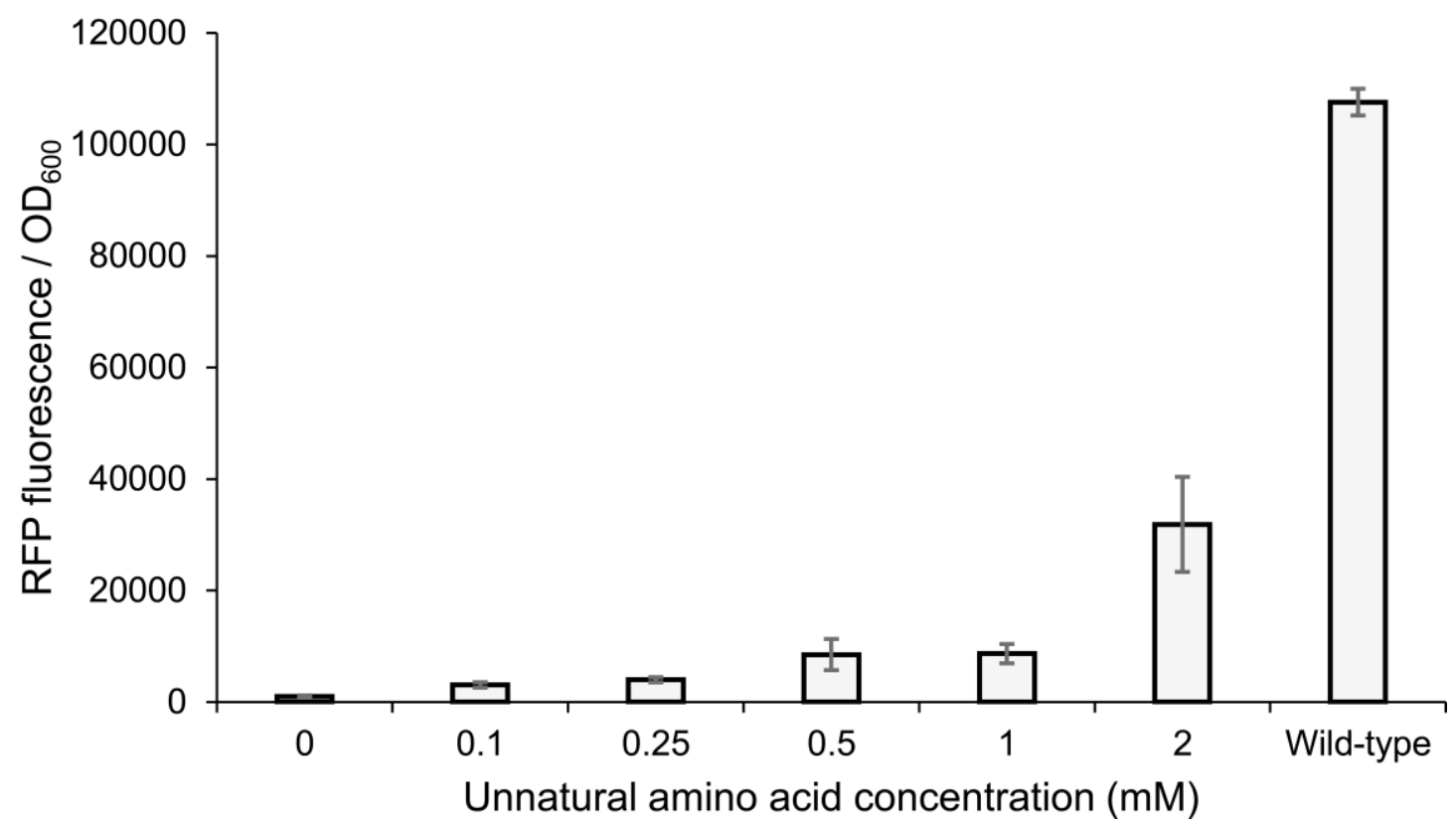

Figure S6. Effect of ncAA concentration on the expression yield of RFP 13X with different cyanopyridylalanine ncAAs. a, mCNP. b, pCNP. RFP fluorescence is normalized by the optical density $\left(\mathrm{OD}_{600}\right)$ and reported as the mean of three biological replicates \pm standard error. Relative to wild-type RFP, 41 and $30 \%$ suppression efficiency were achieved at $1 \mathrm{mM} \mathrm{mCNP}$ and $2 \mathrm{mM}$ pCNP, respectively. 
a

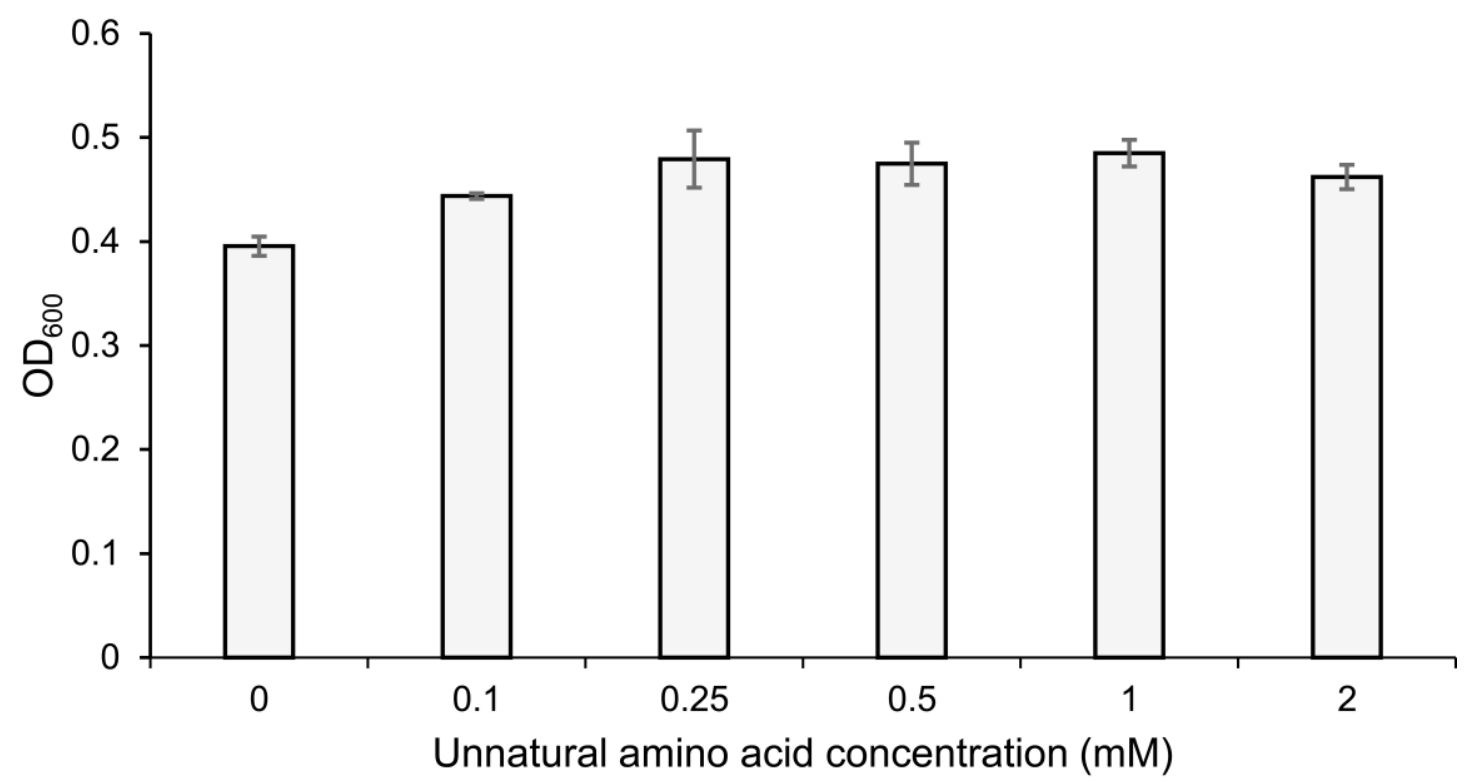

b

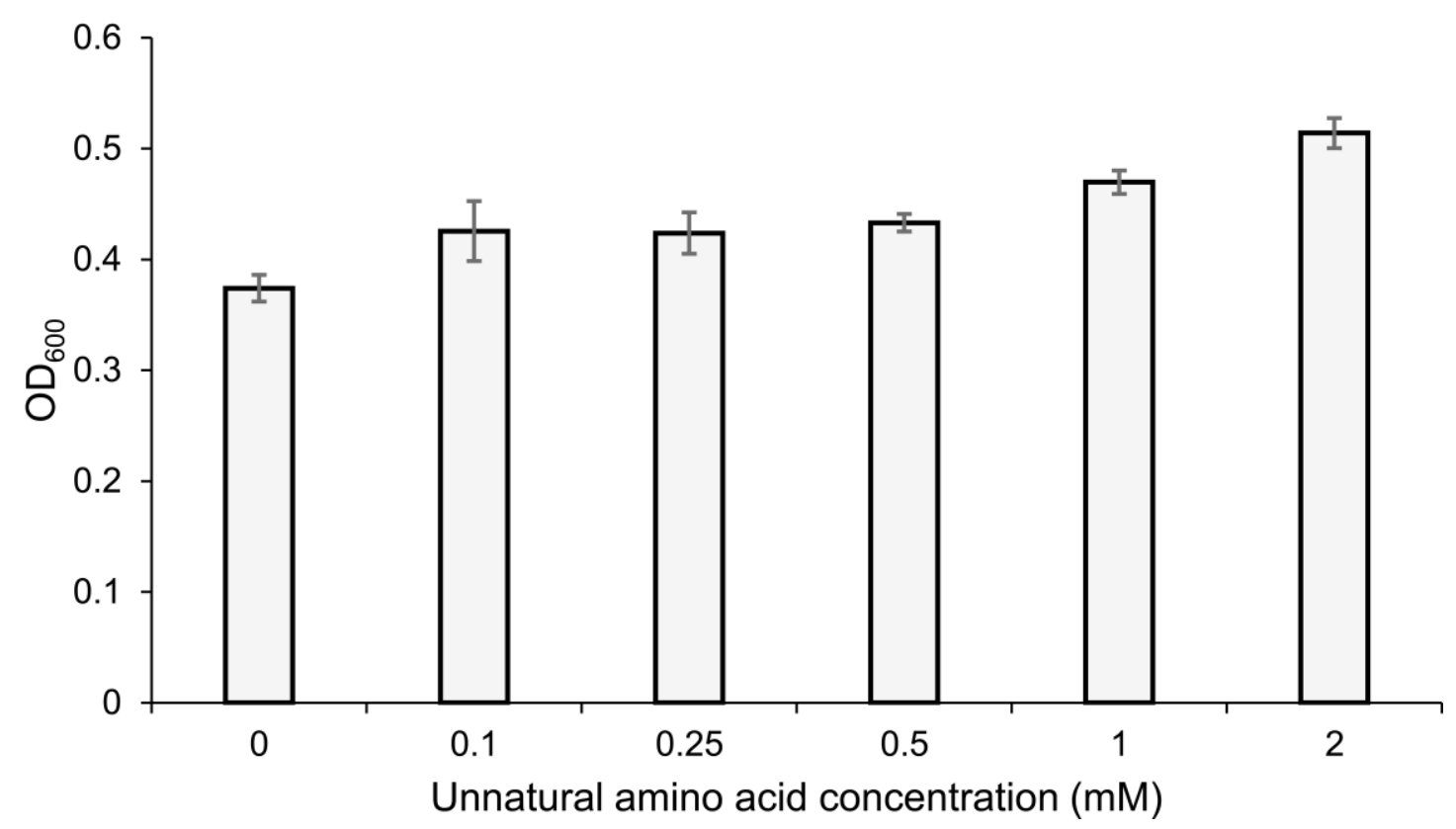

Figure S7. Effect of ncAA concentration on E. coli cell growth. Both mCNP and pCNP are well tolerated by the cells, without any negative effects of cell growth. $\mathrm{OD}_{600}$ values are reported as the mean of three biological replicates \pm standard error measured after incubation with the corresponding ncAA for $16 \mathrm{~h}$ at $25^{\circ} \mathrm{C}$. 
a

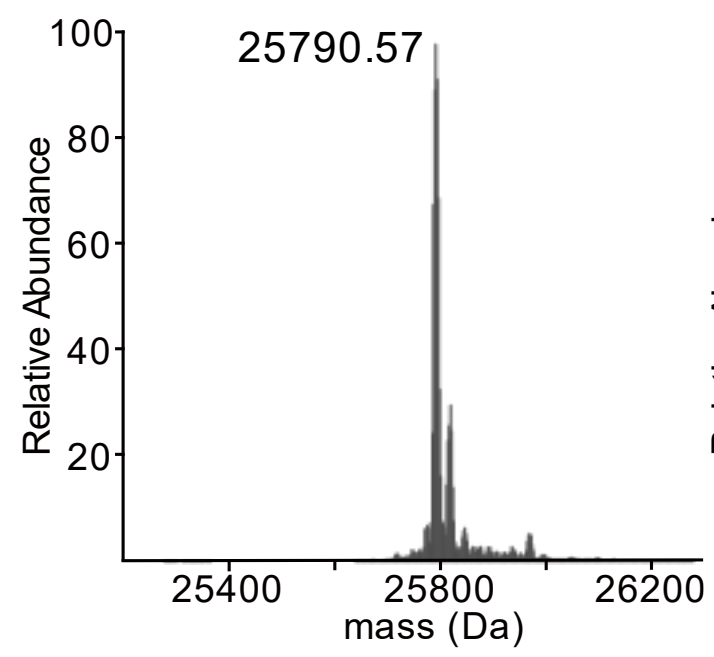

b

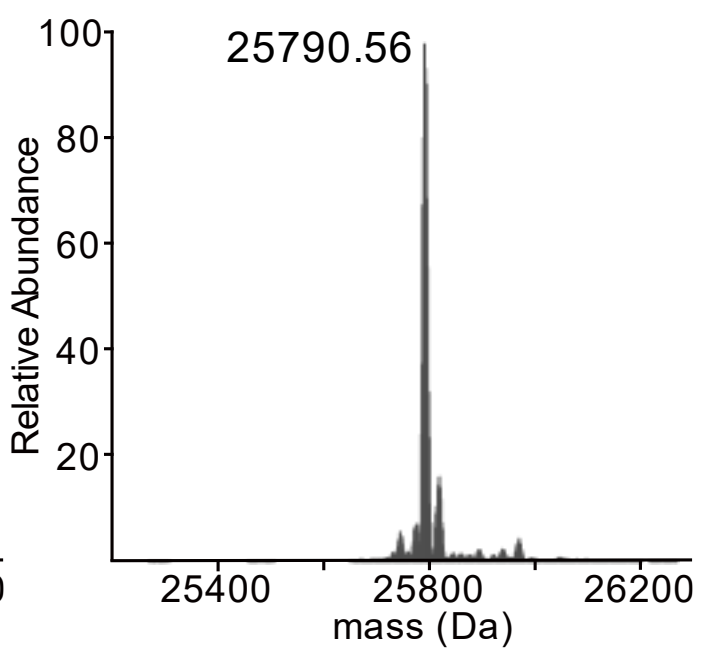

Figure S8. Intact protein mass spectra of the fusion protein NT-Ubi 7X (X indicating the position of the ncAA in the amino acid sequence) showing the high incorporation yield of cyanopyridylalanines. a) NT-Ubi $7 \mathrm{mCNP}$, b) NT-Ubi $7 \mathrm{pCNP}$. The calculated mass minus the $\mathrm{N}$-terminal methionine is $25791.91 \mathrm{Da}$. The minor peak at 25817.57 Da is likely from formylation in the N-terminal NT domain, because after TEV protease cleavage this modification is not observed in the mass spectra of Cys-Ubi-X7. 


\section{Supplementary Tables}

Table S1. Mutations found in the 7 selected G1PylRS variants that recognize mCNP. The selected mutation set in grey indicates the variant used for later applications.

\begin{tabular}{lccccccc} 
Mutant & \multicolumn{7}{c}{ Site } \\
\hline MmPylRS wt & L305 & Y306 & N346 & C348 & Y384 & V401 & W417 \\
\hline G1PylRS wt & L124 & Y125 & N165 & V167 & Y204 & A221 & W237 \\
\hline G1mCNP11 & R & C & S & C & W & A & W \\
\hline G1mCNP13 & G & R & N & V & W & C & W \\
\hline G1mCNP16 & A & L & N & A & W & S & W \\
\hline G1mCNP34 & A & L & N & A & W & S & W \\
\hline G1mCNP43 & A & F & N & V & F & S & Y \\
\hline G1mCNP58 & A & L & N & C & W & S & W \\
\hline G1mCNP60 & A & M & N & S & W & C & W \\
\hline
\end{tabular}


Table S2. Mutations found in the 17 selected G1PylRS variants that recognize pCNP. The selected mutation set in grey indicates the variant used for later applications.

\begin{tabular}{|c|c|c|c|c|c|c|c|}
\hline Mutant & & & & \multicolumn{2}{|l|}{ - Site } & \multirow[b]{2}{*}{ V401 } & \multirow[b]{2}{*}{ W417 } \\
\hline MmPylRS wt & L305 & Y306 & N346 & C348 & Y384 & & \\
\hline G1PylRS wt & L124 & Y125 & N165 & V167 & Y204 & A221 & W237 \\
\hline G1pCNP01 & $\mathrm{A}$ & $\mathrm{F}$ & $\mathrm{N}$ & $\mathrm{V}$ & $\mathrm{W}$ & $\mathrm{A}$ & $\mathrm{Y}$ \\
\hline G1pCNP02 & $\mathrm{S}$ & $\mathrm{F}$ & $\mathrm{N}$ & $\mathrm{V}$ & $\mathrm{W}$ & $S$ & $\mathrm{Y}$ \\
\hline G1pCNP03 & A & $\mathrm{F}$ & $\mathrm{N}$ & $\mathrm{V}$ & $\mathrm{W}$ & $S$ & $\mathrm{Y}$ \\
\hline G1pCNP04 & A & $\mathrm{F}$ & $\mathrm{N}$ & $\mathrm{V}$ & $\mathrm{W}$ & $\mathrm{S}$ & Y \\
\hline G1pCNP05 & A & $\mathrm{F}$ & $\mathrm{N}$ & $\mathrm{V}$ & $\mathrm{W}$ & $\mathrm{S}$ & $\mathrm{Y}$ \\
\hline G1pCNP06 & A & $\mathrm{F}$ & $\mathrm{N}$ & V & $\mathrm{W}$ & A & $\mathrm{Y}$ \\
\hline G1pCNP07 & A & $\mathrm{F}$ & $\mathrm{N}$ & $\mathrm{V}$ & $\mathrm{W}$ & A & Y \\
\hline G1pCNP08 & A & $\mathrm{F}$ & $\mathrm{N}$ & $\mathrm{V}$ & $\mathrm{W}$ & A & Y \\
\hline G1pCNP09 & $\mathrm{A}$ & $\mathrm{F}$ & $\mathrm{N}$ & $\mathrm{V}$ & $\mathrm{W}$ & $S$ & $\mathrm{Y}$ \\
\hline G1pCNP11 & A & $\mathrm{F}$ & $\mathrm{N}$ & $\mathrm{V}$ & W & A & $\mathrm{Y}$ \\
\hline G1pCNP12 & $\mathrm{A}$ & $\mathrm{F}$ & $\mathrm{N}$ & $\mathrm{V}$ & W & S & Y \\
\hline G1pCNP16 & A & $\mathrm{F}$ & $\mathrm{N}$ & $\mathrm{V}$ & W & $\mathrm{A}$ & Y \\
\hline G1pCNP26 & A & $\mathrm{F}$ & $\mathrm{N}$ & $\mathrm{V}$ & $\mathrm{W}$ & A & $\mathrm{Y}$ \\
\hline G1pCNP37 & A & $\mathrm{F}$ & $\mathrm{N}$ & $\mathrm{V}$ & $\mathrm{W}$ & S & Y \\
\hline G1pCNP42 & A & $\mathrm{F}$ & $\mathrm{N}$ & $\mathrm{V}$ & $\mathrm{W}$ & $\mathrm{S}$ & Y \\
\hline G1pCNP43 & $\mathrm{A}$ & $\mathrm{F}$ & $\mathrm{N}$ & $\mathrm{V}$ & $\mathrm{W}$ & $\mathrm{A}$ & $\mathrm{Y}$ \\
\hline G1pCNP58 & A & $\mathrm{F}$ & $\mathrm{N}$ & $\mathrm{V}$ & $\mathrm{W}$ & $\mathrm{S}$ & $\mathrm{Y}$ \\
\hline
\end{tabular}


Table S3. Yields of proteins expressed in the present study reported as mg per $1 \mathrm{~L}$ cell culture $^{\mathrm{a}}$.

\begin{tabular}{lll} 
Protein $^{\text {b }}$ & mCNP & pCNP \\
\hline RFP 13X & 115 & 75 \\
\hline RFP 237X/243X & 69 & N/A \\
\hline RFP 204X/237X & 70 & N/A \\
\hline GB1 11X & 92 & 25 \\
\hline GB1 24X/28X & 68 & 16 \\
\hline Ppib 147X & 100 & 23 \\
\hline NT-Ubi 7X & 256 & 162 \\
\hline Cys-Ubi 7X & 134 & 60
\end{tabular}

${ }^{a}$ Yields are calculated based on the expression of $50 \mathrm{~mL}$ cell culture.

${ }^{\mathrm{b}} \mathrm{X}$ indicates the position of the ncAA (either mCNP or pCNP). 
Table S4. Expected mass of the proteins reported in the present study.

\begin{tabular}{ll} 
Protein & Expected mass (Da) \\
\hline RFP 13X $\left(-\right.$ Met $\left.^{\mathrm{b}}\right)$ & 26714.06 \\
\hline RFP 13X + cysteine & 26818.19 \\
\hline RFP 237X/243X (-Met) & 27504.01 \\
\hline RFP 237X/243X + EDDC & $27736.33^{\mathrm{c}}$ \\
\hline RFP 204X/237X (-Met) & 26876.26 \\
\hline RFP 204X/237X + EDDC & $27108.58^{\mathrm{c}}$ \\
\hline GB1 11X (-Met) & 8390.17 \\
\hline GB1 11X + cysteine & 8494.30 \\
\hline GB1 24X/28X (-Met) & 8465.19 \\
\hline GB1 24X/28X + EDDC & $8697.51^{\mathrm{c}}$ \\
\hline Ppib 147X & 19012.40 \\
\hline Ppib 147X + cysteine & 19116.53 \\
\hline Linear Cys-Ubi 7X & 10519.94 \\
\hline
\end{tabular}

${ }^{a} \mathrm{X}$ indicates the position of the ncAA (either mCNP or pCNP).

$\mathrm{b}$-Met indicates loss of the N-terminal methionine during protein expression.

${ }^{c}$ Expected mass of the cyclization product from the reaction with EDDC. 
Table S5. Complete nucleotide sequences of the plasmids used in the current study.*

\begin{tabular}{|c|c|}
\hline Plasmid & DNA sequence \\
\hline \multirow[t]{43}{*}{ pBK-G1RS } & ATGAGCCATATTCAACGGGAAACGTCTTGCTCGAGGCCGCGATTAAATTCCAACATGGATGCTGAT \\
\hline & TTATATGGGTATAAATGGGCTCGCGATAATGTCGGGCAATCAGGTGCGACAATCTATCGATTGTAT \\
\hline & GGGAAGCCCGATGCGCCAGAGTTGTTTCTGAAACATGGCAAAGGTAGCGTTGCCAATGATGTTACA \\
\hline & GATGAGATGGTCAGACTAAACTGGCTGACGGAATTTATGCCTCTTCCGACCATCAAGCATTTTATC \\
\hline & CGTACTCCTGATGATGCATGGTTACTCACCACTGCGATCCCCGGGAAAACAGCATTCCAGGTATTA \\
\hline & GAAGAATATCCTGATTCAGGTGAAAATATTGTTGATGCGCTGGCAGTGTTCCTGCGCCGGTTGCAT \\
\hline & TCGATTCCTGTTTGTAATTGTCCTTTTAACAGCGATCGCGTATTTCGTCTCGCTCAGGCGCAATCA \\
\hline & CGAATGAATAACGGTTTGGTTGATGCGAGTGATTTTGATGACGAGCGTAATGGCTGGCCTGTTGAA \\
\hline & CAAGTCTGGAAAGAAATGCATAAGCTTTTGCCATTCTCACCGGATTCAGTCGTCACTCATGGTGAT \\
\hline & TTCTCACTTGATAACCTTATTTTTGACGAGGGGAAATTAATAGGTTGTATTGATGTTGGACGAGTC \\
\hline & GGAATCGCAGACCGATACCAGGATCTTGCCATCCTATGGAACTGCCTCGGTGAGTTTTCTCCTTCA \\
\hline & TTACAGAAACGGCTTTTTCAAAAATATGGTATTGATAATCCTGATATGAATAAATTGCAGTTTCAT \\
\hline & TTGATGCTCGATGAGTTTTTCTAACACTGGCAGAGCATTACGCTGACTTGGAGGATCTAGGTGAAG \\
\hline & ATCCTTGGTACGGCCGCCAAGCTTAAAAAAAATCCTTAGCTTTCGCTAAGGATCTGCAGTGGCGAA \\
\hline & AGGCCGGGGGGTCGAACCCCGCTTACAGGTTTTAGAGACCCGTTTGCTCGCCGGAGCGCCCTCCGA \\
\hline & ATTCAGCGTTACAAGTATTACACAAAGTTTTTTATGTTGAGAATATTTTTTTGATGTCCTCGGGTT \\
\hline & GTCAGCCTGTCCCGCTTTAATATCATACGCCGTTATACGTTGTTTACGCTTTGAGGAGGATCCATA \\
\hline & TGGTGGTGAAATTTACCGATAGCCAGATTCAGCATCTGATGGAATATGGTGATAATGATTGGAGCG \\
\hline & AAGCCGAATTTGAAGATGCAGCAGCACGTGATAAAGAATTTAGCAGCCAGTTTAGCAAACTGAAAA \\
\hline & GCGCCAATGATAAAGGCCTGAAAGATGTTATTGCAAATCCGCGTAATGATCTGACCGATCTGGAAA \\
\hline & ACAAAATTCGCGAAAAACTGGCAGCCCGTGGTTTTATTGAAGTTCATACCCCGATTTTTGTGAGCA \\
\hline & AAAGCGCACTGGCAAAAATGACCATTACCGAAGATCATCCGCTGTTCAAACAGGTGTTTTGGATTG \\
\hline & ATGATAAACGTGCACTGCGTCCGATGCATGCAATGAATCTGTATAAAGTTATGCGTGAACTGCGCG \\
\hline & ATCATACCAAAGGTCCGGTTAAAATCTTTGAAATTGGTAGCTGCTTTCGCAAAGAAAGCAAAAGCA \\
\hline & GTACCCATCTGGAAGAATTTACCATGCTGAACCTGGTTGAAATGGGTCCTGATGGTGATCCGATGG \\
\hline & AACATCTGAAAATGTATATTGGCGATATCATGGATGCCGTTGGTGTTGAATATACCACCAGTCGTG \\
\hline & AAGAATCAGATGTTTATGTTGAAACCCTGGACGTGGAAATTAATGGCACCGAAGTTGCAAGCGGTG \\
\hline & CAGTTGGTCCGCATAAACTGGATCCGGCACATGATGTGCATGAACCGTGGGCAGGTATTGGTTTTG \\
\hline & $\overline{\text { GTCTGGAACGTCTGCTGATGCTGAAAAATGGTAAAAGCAATGCACGCAAAACCGGCAAAAGTATTA }}$ \\
\hline & CCTATCTGAATGGCTACAAACTGGATTAACTGCGTCGACGTCTTGGTTTAGAAACAGCAAACAATC \\
\hline & 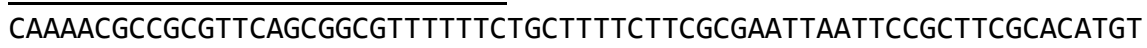 \\
\hline & GAGCAAAAGGCCAGCAAAAGGCCAGGAACCGTAAAAAGGCCGCGTTGCTGGCGTTTTTCCATAGGC \\
\hline & TCCGCCCCCCTGACGAGCATCACAAAAATCGACGCTCAAGTCAGAGGTGGCGAAACCCGACAGGAC \\
\hline & TATAAAGATACCAGGCGTTTCCCCCTGGAAGCTCCCTCGTGCGCTCTCCTGTTCCGACCCTGCCGC \\
\hline & TTACCGGATACCTGTCCGCCTTTCTCCCTTCGGGAAGCGTGGCGCTTTCTCATAGCTCACGCTGTA \\
\hline & GGTATCTCAGTTCGGTGTAGGTCGTTCGCTCCAAGCTGGGCTGTGTGCACGAACCCCCCGTTCAGC \\
\hline & CCGACCGCTGCGCCTTATCCGGTAACTATCGTCTTGAGTCCAACCCGGTAAGACACGACTTATCGC \\
\hline & CACTGGCAGCAGCCACTGGTAACAGGATTAGCAGAGCGAGGTATGTAGGCGGTGCTACAGAGTTCT \\
\hline & TGAAGTGGTGGCCTAACTACGGCTACACTAGAAAGGACAGTATTTGGTATCTGCGCTCTGCTGAAG \\
\hline & CCAGTTACCTTTCGGAAAAAGAGTTGGGTAGCTCTTGATCCGGCAAACAGCCACCGCTGGTAGCGG \\
\hline & TGGTTTTTTTGTTTGCAGCAGCAGATTACACGCAGAAAAAAAGGATCTCAGAAGATCCTTTGATCT \\
\hline & TTTCTACGGGTCTGACGCTCAGTGGAACGAAAACTCACGTTAAGGGATTTTGGTCATGAACAATAA \\
\hline & AACTGTCTGCTTACATAAACAGTAATACAAGGGGTGT \\
\hline
\end{tabular}

pRSF-G1mCNPRS TAATACGACTCACTATAGGGAGACCACAACGGTTTCCCTCTAGAAATAATTTTGTTTAACTTTAAG AAGGAGATATACATATGGTGGTGAAATTTACCGATAGCCAGATTCAGCATCTGATGGAATATGGTG ATAATGATTGGAGCGAAGCCGAATTTGAAGATGCAGCAGCACGTGATAAAGAATTTAGCAGCCAGT ITAGCAAACTGAAAAGCGCCAATGATAAAGGCCTGAAAGATGTTATTGCAAATCCGCGTAATGATC TGACCGATCTGGAAAACAAAATTCGCGAAAAACTGGCAGCCCGTGGTTTTATTGAAGTTCATACCC CGATTTTTGTGAGCAAAAGCGCACTGGCAAAAATGACCATTACCGAAGATCATCCGCTGTTCAAAC AGGTGTTTTGGATTGATGATAAACGTGCACTGCGTCCGATGCATGCAATGAATGCTTTGAAAGTTA TGCGTGAACTGCGCGATCATACCAAAGGTCCGGTTAAAATCTTTGAAATTGGTAGCTGCTTTCGCA AAGAAAGCAAAAGCAGTACCCATCTGGAAGAATTTACCATGCTGAACCTGGCAGAAATGGGTCCTG ATGGTGATCCGATGGAACATCTGAAAATGTATATTGGCGATATCATGGATGCCGTTGGTGTTGAAT ATACCACCAGTCGTGAAGAATCAGATGTTTGGGTTGAAACCCTGGACGTGGAAATTAATGGCACCG 
AAGTTGCAAGCGGTTCTGTTGGTCCGCATAAACTGGATCCGGCACATGATGTGCATGAACCGTGGG

CAGGTATTGGTTTTGGTCTGGAACGTCTGCTGATGCTGAAAAATGGTAAAAGCAATGCACGCAAAA CCGGCAAAAGTATTACCTATCTGAATGGCTACAAACTGGATTAAGAATTCGAGCTCCCGGGTACCA GATAGATCCGGCTGCTAACAAAGCCCGAAAGGAAGCTGAGTTGGCTGCTGCCACCGCTGAGCAATA ACTAGCATAACCCCTTGGGGCCTCTAAACGGGTCTTGAGGGGTTTTTTGGTTTGTGAGCTCCCGTA ATTCCGCTTCGCAACATGTGAGCACCGGTTTATTGACTACCGGAAGCAGTGTGACCGTGTGCTTCT CAAATGCCTGAGGCCAGTTTGCTCAGGCTCTCCCCCGTGGAGGTAATAATTGACGATATGATCAGTG CACGGCTAACTAAGCGGCCTGCTGACTTTCTCGCCGATCAAAAGGCATTTTGCTATTAAGGGATTG ACGAGGGCGTATCTGCGCAGTAAGATGCGCCCCGGATTGGAGGGCGCTCCGGCGAGCAAACGGGTC TCTAAAACCTGTAAGCGGGGTTCGACCCCCCGGCCTTTCGCCAAATTCGAAAAGCCTGCTCAACGA GCAGGCTTTTTTGCATGCTCGAGCAGCTCAGGGTCGAATTTGCTTTCGAATTTTCTGCCATTCATCC GCTTATTATCACTTATTCAGGCGTAGCAACCAGGCGTTTAAGGGCACCAATAACTGCCTTAAAAAA ACTTCCGCTTCCTCGCTCACTGACTCGCTACGCTCGGTCGTTCGACTGCGGCGAGCGGTGTCAGCT CACTCAAAAGCGGTAATACGGTTATCCACAGAATCAGGGGATAAAGCCGGAAAGAACATGTGAGCA AAAAGCAAAGCACCGGAAGAAGCCAACGCCGCAGGCGTTTTTCCATAGGCTCCGCCCCCCTGACGA GCATCACAAAAATCGACGCTCAAGCCAGAGGTGGCGAAACCCGACAGGACTATAAAGATACCAGGC GTTTCCCCCTGGAAGCTCCCTCGTGCGCTCTCCTGTTCCGACCCTGCCGCTTACCGGATACCTGTC CGCCTTTCTCCCTTCGGGAAGCGTGGCGCTTTCTCATAGCTCACGCTGTTGGTATCTCAGTTCGGT GTAGGTCGTTCGCTCCAAGCTGGGCTGTGTGCACGAACCCCCCGTTCAGCCCGACCGGCTGCGCCTT ATCCGGTAACTATCGTCTTGAGTCCAACCCGGTAAGACACGACTTATCGCCACTGGCAGCAGCCAT TGGTAACTGATTTAGAGGACTTTGTCTTGAAGTTATGCACCTGTTAAGGCTAAACTGAAAGAACAG ATTTTGGTGAGTGCGGTCCTCCAACCCACTTACCTTGGTTCAAAGAGTTGGTAGCTCAGCGAACCT TGAGAAAACCACCGTTGGTAGCGGTGGTTTTTCTTTAATTTATGAGATGATGAATCAATCGGTCTAT CAAGTCAACGAACAGCTATTCCGTTGAACTGTGGTACGCGTTAGAAAAACTCATCGAGCATCAAAT GAAACTGCAATTTATTCATATCAGGATTATCAATACCATATTTTTGAAAAAGCCGTTTCTGTAATG AAGGAGAAAACTCACCGAGGCAGTTCCATAGGATGGCAAGATCCTGGTATCGGTCTGCGATTCCGA CTCGTCCAACATCAATACAACCTATTAATTTCCCCTCGTCAAAAATAAGGTTATCAAGTGAGAAAT CACCATGAGTGACGACTGAATCCGGTGAGAATGGCAAAAGTTTATGCATTTCTTTCCAGACTTGTT CAACAGGCCAGCCATTACGCTCGTCATCAAAATCACTCGCATCAACCAAACCGTTATTCATTCGTG ATTGCGCCTGAGCGAGACGAAATACGCGGTCGCTGTTAAAAGGACAATTACAAACAGGAATCGAAT GCAACCGGCGCAGGAACACTGCCAGCGCATCAACAATATTTTCACCTGAATCAGGATATTCTTCTA ATACCTGGAATGCTGTTTTCCCGGGGATCGCAGTGGTGAGTAACCATGCATCATCAGGAGTACGGA TAAAATGCTTGATGGTCGGAAGAGGCATAAATTCCGTCAGCCAGTTTAGTCTGACCATCTCATCTG TAACATCATTGGCAACGCTACCTTTGCCATGTTTCAGAAACAACTCTGGCGCATCGGGCTTCCCAT ACAATCGATAGATTGTCGCACCTGATTGCCCGACATTATCGCGAGCCCATTTATACCCATATAAAT CAGCATCCATGTTGGAATTTAATCGCGGCCTAGAGCAAGACGTTTCCCGTTGAATATGGCTCATAC TCTTCCTTTTTCAATATTATTGAAGCATTTATCAGGGTTATTGTCTCATGAGCGGATACATATTTG AATGTATTTAGAAAAATAAACAAATAGGCATGCAGCGCTTGACTTGACGGGACGGCGTTGTAATTC TCATGTTTGACAGCTTATCATCGATAAGCTTGGTACCCAA

pRSF-G1pCNPRS

TAATACGACTCACTATAGGGAGACCACAACGGTTTCCCTCTAGAAATAATTTTGTTTAACTTTAAG AAGGAGATATACATATGGTGGTGAAATTTACCGATAGCCAGATTCAGCATCTGATGGAATATGGTG ATAATGATTGGAGCGAAGCCGAATTTGAAGATGCAGCAGCACGTGATAAAGAATTTAGCAGCCAGT TTAGCAAACTGAAAAGCGCCAATGATAAAGGCCTGAAAGATGTTATTGCAAATCCGCGTAATGATC TGACCGATCTGGAAAACAAAATTCGCGAAAAACTGGCAGCCCGTGGTTTTATTGAAGTTCATACCC CGATTTTTGTGAGCAAAAGCGCACTGGCAAAAATGACCATTACCGAAGATCATCCGCTGTTCAAAC AGGTGTTTTGGATTGATGATAAACGTGCACTGCGTCCGATGCATGCAATGAATGCTTTTAAAGTTA TGCGTGAACTGCGCGATCATACCAAAGGTCCGGTTAAAATCTTTGAAATTGGTAGCTGCTTTCGCA AAGAAAGCAAAAGCAGTACCCATCTGGAAGAATTTACCATGCTGAACCTGGTAGAAATGGGTCCTG ATGGTGATCCGATGGAACATCTGAAAATGTATATTGGCGATATCATGGATGCCGTTGGTGTTGAAT ATACCACCAGTCGTGAAGAATCAGATGTTTGGGTTGAAACCCTGGACGTGGAAATTAATGGCACCG AAGTTGCAAGCGGTTCTGTTGGTCCGCATAAACTGGATCCGGCACATGATGTGCATGAACCGTATG CAGGTATTGGTTTTGGTCTGGAACGTCTGCTGATGCTGAAAAATGGTAAAAGCAATGCACGCAAAA CCGGCAAAAGTATTACCTATCTGAATGGCTACAAACTGGATTAAGAATTCGAGCTCCCGGGTACCA GATAGATCCGGCTGCTAACAAAGCCCGAAAGGAAGCTGAGTTGGCTGCTGCCACCGCTGAGCAATA ACTAGCATAACCCCTTGGGGCCTCTAAACGGGTCTTGAGGGGTTTTTTGGTTTGTGAGCTCCCGTA ATTCCGCTTCGCAACATGTGAGCACCGGTTTATTGACTACCGGAAGCAGTGTGACCGTGTGCTTCT CAAATGCCTGAGGCCAGTTTGCTCAGGCTCTCCCCGTGGAGGTAATAATTGACGATATGATCAGTG CACGGCTAACTAAGCGGCCTGCTGACTTTCTCGCCGATCAAAAGGCATTTTGCTATTAAGGGATTG ACGAGGGCGTATCTGCGCAGTAAGATGCGCCCCGCATTGGAGGGCGCTCCGGCGAGCAAACGGGTC TCTAAAACCTGTAAGCGGGGTTCGACCCCCCGGCCTTTCGCCAAATTCGAAAAGCCTGCTCAACGA 
GCAGGCTTTTTTGCATGCTCGAGCAGCTCAGGGTCGAATTTGCTTTCGAATTTCTGCCATTCATCC GCTTATTATCACTTATTCAGGCGTAGCAACCAGGCGTTTAAGGGCACCAATAACTGCCTTAAAAAA ACTTCCGCTTCCTCGCTCACTGACTCGCTACGCTCGGTCGTTCGACTGCGGCGAGCGGTGTCAGCT CACTCAAAAGCGGTAATACGGTTATCCACAGAATCAGGGGATAAAGCCGGAAAGAACATGTGAGCA AAAAGCAAAGCACCGGAAGAAGCCAACGCCGCAGGCGTTTTTCCATAGGCTCCGCCCCCCTGACGA GCATCACAAAAATCGACGCTCAAGCCAGAGGTGGCGAAACCCGACAGGACTATAAAGATACCAGGC GTTTCCCCCTGGAAGCTCCCTCGTGCGCTCTCCTGTTCCGACCCTGCCGCTTACCGGATACCTGTC CGCCTTTCTCCCTTCGGGAAGCGTGGCGCTTTCTCATAGCTCACGCTGTTGGTATCTCAGTTCGGT GTAGGTCGTTCGCTCCAAGCTGGGCTGTGTGCACGAACCCCCCGTTCAGCCCGACCGCTGCGCCTT ATCCGGTAACTATCGTCTTGAGTCCAACCCGGTAAGACACGACTTATCGCCACTGGCAGCAGCCAT TGGTAACTGATTTAGAGGACTTTGTCTTGAAGTTATGCACCTGTTAAGGCTAAACTGAAAGAACAG ATTTTGGTGAGTGCGGTCCTCCAACCCACTTACCTTGGTTCAAAGAGTTGGTAGCTCAGCGAACCT TGAGAAAACCACCGTTGGTAGCGGTGGTTTTTCTTTATTTATGAGATGATGAATCAATCGGTCTAT CAAGTCAACGAACAGCTATTCCGTTGAACTGTGGTACGCGTTAGAAAAACTCATCGAGCATCAAAT GAAACTGCAATTTATTCATATCAGGATTATCAATACCATATTTTTGAAAAAGCCGTTTCTGTAATG AAGGAGAAAACTCACCGAGGCAGTTCCATAGGATGGCAAGATCCTGGTATCGGTCTGCGATTCCGA CTCGTCCAACATCAATACAACCTATTAATTTCCCCTCGTCAAAAATAAGGTTATCAAGTGAGAAAT CACCATGAGTGACGACTGAATCCGGTGAGAATGGCAAAAGTTTATGCATTTCTTTCCAGACTTGTT CAACAGGCCAGCCATTACGCTCGTCATCAAAATCACTCGCATCAACCAAACCGTTATTCATTCGTG ATTGCGCCTGAGCGAGACGAAATACGCGGTCGCTGTTAAAAGGACAATTACAAACAGGAATCGAAT GCAACCGGCGCAGGAACACTGCCAGCGCATCAACAATATTTTCACCTGAATCAGGATATTCTTCTA ATACCTGGAATGCTGTTTTCCCGGGGATCGCAGTGGTGAGTAACCATGCATCATCAGGAGTACGGA TAAAATGCTTGATGGTCGGAAGAGGCATAAATTCCGTCAGCCAGTTTAGTCTGACCATCTCATCTG TAACATCATTGGCAACGCTACCTTTGCCATGTTTCAGAAACAACTCTGGCGCATCGGGCTTCCCAT ACAATCGATAGATTGTCGCACCTGATTGCCCGACATTATCGCGAGCCCATTTATACCCATATAAAT CAGCATCCATGTTGGAATTTAATCGCGGCCTAGAGCAAGACGTTTCCCGTTGAATATGGCTCATAC TCTTCCTTTTTCAATATTATTGAAGCATTTATCAGGGTTATTGTCTCATGAGCGGATACATATTTG AATGTATTTAGAAAAATAAACAAATAGGCATGCAGCGCTTGACTTGACGGGACGGCGTTGTAATTC TCATGTTTGACAGCTTATCATCGATAAGCTTGGTACCCAA

pCDF-PpiB147TAG TAATACGACTCACTATAGGGAGACCACAACGGTTTCCCTCTAGAAATAATTTTGTTTAACTTTAAG AAGGAGATATACATATGGTTACTTTCCACACCAATCACGGCGATATTGTCATCAAAACTTTTGACG ATAAAGCACCTGAAACAGTTAAAAACTTCCTGGACTACTGCCGCGAAGGTTTTTACAACAACACCA TTTTCCACCGTGTTATCAACGGCTTTATGATTCAGGGCGGCGGTTTTGAACCGGGCATGAAACAAA AAGCCACCAAAGAACCGATCAAAAACGAAGCCAACAACGGCCTGAAAAATACCCGTGGTACGCTGG CAATGGCACGTACTCAGGCTCCGCACTCTGCAACTGCACAGTTCTTCATCAACGTGGTTGATAACG ACTTCCTGAACTTCTCTGGCGAAAGCCTGCAAGGTTGGGGCTACTGCGTGTTTGCTGAAGTGGTTG ACGGCATGGACGTGGTAGACAAAATCAAAGGTGTTGCAACCGGTCGTAGCGGTATGTAGCAGGACG TGCCAAAAGAAGACGTTATCATTGAAAGCGTGACCGTTAGCGAGCACCACCATCATCACCACTAAT AAAGAATTCGAGCTCCCGGGTACCATGGCATGCATCGATAGATCCGGCTGCTAACAAAGCCCGAAA GGAAGCTGAGTTGGCTGCTGCCACCGCTGAGCAATAACTAGCATAACCCCTTGGGGCCTCTAAACG GGTCTTGAGGGGTTTTTTGCTGAAAGGAGGAACTACAGGCATTTGAGAAGCACACGGTCACACTGC TTCCGGTAGTCAATAAACCGGTAAACCAGCAATAGACATAAGCGGCTATTTAACGACCCTGCCCTG AACCGACGACCGGGTCATCGTGGCCGGATCTTGCGGCCCCTCGGCTTGAACGAATTGTTAGACATT ATTTGCCGACTACCTTGGTGATCTCGCCTTTCACGTAGTGGACAAATTCTTCCAACTGATCTGCGC GCGAGGCCAAGCGATCTTCTTCTTGTCCAAGATAAGCCTGTCTAGCTTCAAGTATGACGGGCTGAT ACTGGGCCGGCAGGCGCTCCATTGCCCAGTCGGCAGCGACATCCTTCGGCGCGATTTTGCCGGTTA CTGCGCTGTACCAAATGCGGGACAACGTAAGCACTACATTTCGCTCATCGCCAGCCCAGTCGGGCG GCGAGTTCCATAGCGTTAAGGTTTCATTTAGCGCCTCAAATAGATCCTGTTCAGGAACCGGATCAA AGAGTTCCTCCGCCGCTGGACCTACCAAGGCAACGCTATGTTCTCTTGCTTTTGTCAGCAAGATAG CCAGATCAATGTCGATCGTGGCTGGCTCGAAGATACCTGCAAGAATGTCATTGCGCTGCCATTCTC CAAATTGCAGTTCGCGCTTAGCTGGATAACGCCACGGAATGATGTCGTCGTGCACAACAATGGTGA CTTCTACAGCGCGGAGAATCTCGCTCTCTCCAGGGGAAGCCGAAGTTTCCAAAAGGTCGTTGATCA AAGCTCGCCGCGTTGTTTCATCAAGCCTTACGGTCACCGTAACCAGCAAATCAATATCACTGTGTG GCTTCAGGCCGCCATCCACTGCGGAGCCGTACAAATGTACGGCCAGCAACGTCGGTTCGAGATGGC GCTCGATGACGCCAACTACCTCTGATAGTTGAGTCGATACTTCGGCGATCACCGCTTCCCTCATAC TCTTCCTTTTTCAATATTATTGAAGCATTTATCAGGGTTATTGTCTCATGAGCGGATACATATTTG AATGTATTTAGAAAAATAAACAAATAGCTAGCTCACTCGGTCGCTACGCTCCGGGCGTGAGACTGC GGCGGGCGCTGCGGACACATACAAAGTTACCCACAGATTCCGTGGATAAGCAGGGGACTAACATGT GAGGCAAAACAGCAGGGCCGCGCCGGTGGCGTTTTTCCATAGGCTCCGCCCTCCTGCCAGAGTTCA CATAAACAGACGCTTTTCCGGTGCATCTGTGGGAGCCGTGAGGCTCAACCATGAATCTGACAGTAC 
GGGCGAAACCCGACAGGACTTAAAGATCCCCACCGTTTCCGGCGGGTCGCTCCCTCTTGCGCTCTC CTGTTCCGACCCTGCCGTTTACCGGATACCTGTTCCGCCTTTCTCCCTTACGGGAAGTGTGGCGCT TTCTCATAGCTCACACACTGGTATCTCGGCTCGGTGTAGGTCGTTCGCTCCAAGCTGGGCTGTAAG CAAGAACTCCCCGTTCAGCCCGACTGCTGCGCCTTATCCGGTAACTGTTCACTTGAGTCCAACCCG GAAAAGCACGGTAAAACGCCACTGGCAGCAGCCATTGGTAACTGGGAGTTCGCAGAGGATTTGTTT AGCTAAACACGCGGTTGCTCTTGAAGTGTGCGCCAAAGTCCGGCTACACTGGAAGGACAGATTTGG TTGCTGTGCTCTGCGAAAGCCAGTTACCACGGTTAAGCAGTTCCCCAACTGACTTAACCTTCGATC AAACCACCTCCCCAGGTGGTTTTTTCGTTTACAGGGCAAAAGATTACGCGCAGAAAAAAAGGATCT CAAGAAGATCCTTTGATCTTTTCTACTGAACCGCTCTAGATTTCAGTGCAATTTATCTCTTCAAAT GTAGCACCTGAAGTCAGCCCCATACGATATAAGTTGTAATTCTCATGTTAGTCATGCCCCGCGCCC ACCGGAAGGAGCTGACTGGGTTGAAGGCTCTCAAGGGCATCGGTCGAGATCCCGGTGCCTAATGAG TGAGCTAACTTACATTAATTGCGTTGCGCTCACTGCCCGCTTTCCAGTCGGGAAACCTGTCGTGCC AGCTGCATTAATGAATCGGCCAACGCGCGGGGAGAGGCGGTTTGCGTATTGGGCGCCAGGGTGGTT TTTCTTTTCACCAGTGAGACGGGCAACAGCTGATTGCCCTTCACCGCCTGGCCCTGAGAGAGTTGC AGCAAGCGGTCCACGCTGGTTTGCCCCAGCAGGCGAAAATCCTGTTTGATGGTGGTTAACGGCGGG ATATAACATGAGCTGTCTTCGGTATCGTCGTATCCCACTACCGAGATGTCCGCACCAACGCGCAGC CCGGACTCGGTAATGGCGCGCATTGCGCCCAGCGCCATCTGATCGTTGGCAACCAGCATCGCAGTG GGAACGATGCCCTCATTCAGCATTTGCATGGTTTGTTGAAAACCGGACATGGCACTCCAGTCGCCT TCCCGTTCCGCTATCGGCTGAATTTGATTGCGAGTGAGATATTTATGCCAGCCAGCCAGACGCAGA CGCGCCGAGACAGAACTTAATGGGCCCGCTAACAGCGCGATTTGCTGGTGACCCAATGCGACCAGA TGCTCCACGCCCAGTCGCGTACCGTCTTCATGGGAGAAAATAATACTGTTGATGGGTGTCTGGTCA GAGACATCAAGAAATAACGCCGGAACATTAGTGCAGGCAGCTTCCACAGCAATGGCATCCTGGTCA TCCAGCGGATAGTTAATGATCAGCCCACTGACGCGTTGCGCGAGAAGATTGTGCACCGCCGCTTTA CAGGCTTCGACGCCGCTTCGTTCTACCATCGACACCACCACGCTGGCACCCAGTTGATCGGCGCGA GATTTAATCGCCGCGACAATTTGCGACGGCGCGTGCAGGGCCAGACTGGAGGTGGCAACGCCAATC AGCAACGACTGTTTGCCCGCCAGTTGTTGTGCCACGCGGTTGGGAATGTAATTCAGCTCCGCCATC GCCGCTTCCACTTTTTCCCGCGTTTTCGCAGAAACGTGGCTGGCCTGGTTCACCACGCGGGAAACG GTCTGATAAGAGACACCGGCATACTCTGCGACATCGTATAACGTTACTGGTTTCACATTCACCACC CTGAATTGACTCTCTTCCGGGCGCTATCATGCCATACCGCGAAAGGTTTTGCGCCATTCGATGGTG TCCGGGATCTCGACGCTCTCCCTTATGCGACTCCTGCATTAGGAAAT

pBAD-TEV

TAATACGACTCACTATAGGGGAATTGTGAGCGGATAACAATTCCCCTCTAGAAATAATTTTGTTTA ACTTTAAGAAGGAGATATACATATGGGAGAAAGCTTGTTTAAGGGGCCGCGTGATTACAACCCGAT ATCGAGCAGCATTTGTCATTTGACGAATGAATCTGATGGGCACACAACATCGTTGTATGGTATTGG ATTTGGTCCCTTCATCATTACAAACAAGCACTTGTTTAGAAGAAATAATGGAACACTGTTGGTCCA ATCACTACATGGTGTATTCAAGGTCAAGGATACCACGACTTTGCAACAACACCTCGTGGATGGGAG GGACATGATAATTATTCGCATGCCTAAGGATTTCCCACCATTTCCTCAAAAGCTGAAATTTAGAGA GCCACAAAGGGAAGAGCGCATATGTCTTGTGACAACCAACTTCCAAACTAAGAGCATGTCTAGCAT GGTGTCAGACACTAGTTGCACATTCCCTTCATCTGATGGCATATTCTGGAAGCATTGGATTCAAAC CAAGGATGGGCAGTGTGGCAGTCCATTAGTATCAACTAGAGATGGGTTCATTGTTGGTATACACTC AGCATCGAATTTCACCAACACAAACAATTATTTCACAAGCGTGCCGAAAAACTTCATGGAATTGTT GACAAATCAGGAGGCGCAGCAGTGGGTTAGTGGTTGGCGATTAAATGCTGACTCAGTATTGTGGGG GGGCCATAAAGTTTTCATGGTGAAACCTGAAGAGCCTTTTCAGCCAGTTAAGGAAGCGACTCAACT CATGAATTAAGAATTCGAGCTCCCGGGTACCATGGCATGCATCGATAGATCCGGCTGCTAACAAAG CCCGAAAGGAAGCTGAGTTGGCTGCTGCCACCGCTGAGCAATAACTAGCATAACCCCTTGGGGCCT CTAAACGGGTCTTGAGGGGTTTTTTGCTGAAAGGAGTGCGTTTCTACAAACTCTTTTGTTTATTTT TCTAAATACATTCAAATATGTATCCGCTCATGAGACAATAACCCTGATAAATGCTTCAATAATATT GAAAAAGGAAGAGTATGAGTATTCAACATTTCCGTGTCGCCCTTATTCCCTTTTTTGCGGCATTTT GCCTTCCTGTTTTTGCTCACCCAGAAACGCTGGTGAAAGTAAAAGATGCTGAAGATCAGTTGGGTG CACGAGTGGGTTACATCGAACTGGATCTCAACAGCGGTAAGATCCTTGAGAGTTTTCGCCCCGAAG AACGTTTTCCAATGATGAGCACTTTTAAAGTTCTGCTATGTGGCGCGGTATTATCCCGTGTTGACG CCGGGCAAGAGCAACTCGGTCGCCGCATACACTATTCTCAGAATGACTTGGTTGAGTACTCACCAG TCACAGAAAAGCATCTTACGGATGGCATGACAGTAAGAGAATTATGCAGTGCTGCCATAACCATGA GTGATAACACTGCGGCCAACTTACTTCTGACAACGATCGGAGGACCGAAGGAGCTAACCGCTTTTT TGCACAACATGGGGGATCATGTAACTCGCCTTGATCGTTGGGAACCGGAGCTGAATGAAGCCATAC CAAACGACGAGCGTGACACCACGATGCCTGCAGCAATGGCAACAACGTTGCGCAAACTATTAACTG GCGAACTACTTACTCTAGCTTCCCGGCAACAATTAATAGACTGGATGGAGGCGGATAAAGTTGCAG GACCACTTCTGCGCTCGGCCCTTCCGGCTGGCTGGTTTATTGCTGATAAATCTGGAGCCGGTGAGC GTGGGTCTCGCGGTATCATTGCAGCACTGGGGCCAGATGGTAAGCCCTCCCGTATCGTAGTTATCT ACACGACGGGGAGTCAGGCAACTATGGATGAACGAAATAGACAGATCGCTGAGATAGGTGCCTCAC TGATTAAGCATTGGTAACTGTCAGACCAAGTTTACTCATATATACTTTAGATTGATTTACGCGCCC 
TGTAGCGGCGCATTAAGCGCGGCGGGTGTGGTGGTTACGCGCAGCGTGACCGCTACACTTGCCAGC GCCCTAGCGCCCGCTCCTTTCGCTTTCTTCCCTTCCTTTCTCGCCACGTTCGCCGGCTTTCCCCGT CAAGCTCTAAATCGGGGGCTCCCTTTAGGGTTCCGATTTAGTGCTTTACGGCACCTCGACCCCAAA AAACTTGATTTGGGTGATGGTTCACGTAGTGGGCCATCGCCCTGATAGACGGTTTTTCGCCCTTTG ACGTTGGAGTCCACGTTCTTTAATAGTGGACTCTTGTTCCAAACTTGAACAACACTCAACCCTATC TCGGGCTATTCTTTTGATTTATAAGGGATTTTGCCGATTTCGGCCTATTGGTTAAAAAATGAGCTG ATTTAACAAAAATTTAACGCGAATTTTAACAAAATATTAACGTTTACAATTTAAAAGGATCTAGGT GAAGATCCTTTTTGATAATCTCATGACCAAAATCCCTTAACGTGAGTTTTCGTTCCACTGAGCGTC AGACCCCGTAGAAAAGATCAAAGGATCTTCTTGAGATCCTTTTTTTCTGCGCGTAATCTGCTGCTT GCAAACAAAAAAACCACCGCTACCAGCGGTGGTTTGTTTGCCGGATCAAGAGCTACCAACTCTTTT TCCGAAGGTAACTGGCTTCAGCAGAGCGCAGATACCAAATACTGTCCTTCTAGTGTAGCCGTAGTT AGGCCACCACTTCAAGAACTCTGTAGCACCGCCTACATACCTCGCTCTGCTAATCCTGTTACCAGT CAGGCATTTGAGAAGCACACGGTCACACTGCTTCCGGTAGTCAATAAACCGGTAAACCAGCAATAG ACATAAGCGGCTATTTAACGACCCTGCCCTGAACCGACGACCGGGTCGAATTTGCTTTCGAATTTC TGCCATTCATCCGCTTATTATCACTTATTCAGGCGTAGCACCAGGCGTTTAAGGGCACCAATAACT GCCTTAAAAAAATTACGCCCCGCCCTGCCACTCATCGCAGTACTGTTGTAATTCATTAAGCATTCT GCCGACATGGAAGCCATCACAGACGGCATGATGAACCTGAATCGCCAGCGGCATCAGCACCTTGTC GCCTTGCGTATAATATTTGCCGCTAGCGGAGTGTATACTGGCTTACTATGTTGGCACTGATGAGGG TGTCAGTGAAGTGCTTCATGTGGCAGGAGAAAAAAGGCTGCACCGGTGCGTCAGCAGAATATGTGA TACAGGATATATTCCGCTTCCTCGCTCACTGACTCGCTACGCTCGGTCGTTCGACTGCGGCGAGCG GAAATGGCTTACGAACGGGGCGGAGATTTCCTGGAAGATGCCAGGAAGATACTTAACAGGGAAGTG AGAGGGCCGCGGCAAAGCCGTTTTTCCATAGGCTCCGCCCCCCTGACAAGCATCACGAAATCTGAC GCTCAAATCAGTGGTGGCGAAACCCGACAGGACTATAAAGATACCAGGCGTTTCCCCCTGGCGGCT CCCTCGTGCGCTCTCCTGTTCCTGCCTTTCGGTTTACCGGTGTCATTCCGCTGTTATGGCCGCGTT TGTCTCATTCCACGCCTGACACTCAGTTCCGGGTAGGCAGTTCGCTCCAAGCTGGACTGTATGCAC GAACCCCCCGTTCAGTCCGACCGCTGCGCCTTATCCGGTAACTATCGTCTTGAGTCCAACCCGGAA AGACATGCAAAAGCACCACTGGCAGCAGCCACTGGTAATTGATTTAGAGGAGTTAGTCTTGAAGTC ATGCGCCGGTTAAGGCTAAACTGAAAGGACAAGTTTTGGTGACTGCGCTCCTCCAAGCCAGTTACC TCGGTTCAAAGAGTTGGTAGCTCAGAGAACCTTCGAAAAACCGCCCTGCAAGGCGGTTTTTTCGTT TTCAGAGCAAGAGATTACGCGCAGACCAAAACGATCTCAAGAAGATCATCTTATTAATCAGATAAA ATATTTGCTCATGAGCCCGAAGTGGCGAGCCCGATCTTCCCCATCGGTGATGTCGGCGATATAGGC GCCAGCAACCGCACCTGTGGCGCCGGTGATGCCGGCCACGATGCGTCCGGCGTAGAGGATCTGCTC ATGTTTGACAGCTTATCATCGATCAGCTGATAGAAACAGAAGCCACTGGAGCACCTCAAAAACACC ATCATACACTAAATCAGTAAGTTGGCAGCATCACCCGACGCACTTTGCGCCGAATAAATACCTGTG ACGGAAGATCACTTCGCAGAATAAATAAATCCTGGTGTCCCTGTTGATACCGGGAAGCCCTGGGCC AACTTTTGGCGAAAATGAGACGTCAGTTTGCTCAGGCTCTCCCCGTGGAGGTAATAATTGCCCGCG AAAT

* The sequence encoding the protein of interest is underlined. 
Table S6. DNA and corresponding amino acid sequences of the proteins used in the

current study.

Protein

G1RSwt
DNA sequence

ATGGTGGTGAAATTTACCGATAGCCAGATTCAGCATCTGATGGAA TATGGTGATAATGATTGGAGCGAAGCCGAATTTGAAGATGCAGCA GCACGTGATAAAGAATTTAGCAGCCAGTTTAGCAAACTGAAAAGC GCCAATGATAAAGGCCTGAAAGATGTTATTGCAAATCCGCGTAAT GATCTGACCGATCTGGAAAACAAAATTCGCGAAAAACTGGCAGCC CGTGGTTTTATTGAAGTTCATACCCCGATTTTTGTGAGCAAAAGC GCACTGGCAAAAATGACCATTACCGAAGATCATCCGCTGTTCAAA CAGGTGTTTTGGATTGATGATAAACGTGCACTGCGTCCGATGCAT GCAATGAATCTGTATAAAGTTATGCGTGAACTGCGCGATCATACC AAAGGTCCGGTTAAAATCTTTGAAATTGGTAGCTGCTTTCGCAAA GAAAGCAAAAGCAGTACCCATCTGGAAGAATTTACCATGCTGAAC CTGGTTGAAATGGGTCCTGATGGTGATCCGATGGAACATCTGAAA ATGTATATTGGCGATATCATGGATGCCGTTGGTGTTGAATATACC ACCAGTCGTGAAGAATCAGATGTTTATGTTGAAACCCTGGACGTG GAAATTAATGGCACCGAAGTTGCAAGCGGTGCAGTTGGTCCGCAT AAACTGGATCCGGCACATGATGTGCATGAACCGTGGGCAGGTATT GGTTTTGGTCTGGAACGTCTGCTGATGCTGAAAAATGGTAAAAGC AATGCACGCAAAACCGGCAAAAGTATTACCTATCTGAATGGCTAC AAACTGGATTAA

RFP 13X

RFP $237 X / 243 X$
ATGGCTTCTATGACCGGTCATCACCATCACCATCACTAGGCCAGT AGTGAAGACGTTATCAAGGAGTTTATGCGTTTCAAAGTACGTATG GAGGGTAGTGTTAACGGACACGAATTTGAGATCGAGGGAGAGGGG GAAGGTCGTCCTTACGAGGGAACTCAAACGGCCAAATTAAAGGTG ACCAAAGGTGGGCCCTTGCCATTCGCGTGGGACATCTTGTCACCC CAGTTCCAGTACGGGTCGAAGGCATACGTAAAACACCCAGCGGAC ATTCCTGACTATCTTAAGTTATCTTTCCCGGAAGGTTTTAAATGG GAACGCGTGATGAACTTTGAGGATGGGGGGGTTGTTACGGTGACA CAAGACTCCTCATTGCAAGATGGAGAGTTTATCTATAAAGTCAAA CTTCGCGGCACCAATTTTCCATCTGACGGTCCTGTAATGCAGAAA AAAACAATGGGCTGGGAAGCCTCCACAGAACGTATGTACCCCGAA GATGGAGCTTTAAAGGGCGAAATTAAAATGCGCTTAAAACTTAAA GACGGCGGCCATTACGACGCCGAAGTGAAAACGACGTATATGGCT AAGAAACCCGTCCAGCTTCCGGGAGCCTATAAAACTGACATCAAA CTGGATATTACATCACACAACGAAGATTATACTATTGTCGAACAG TACGAACGCGCCGAAGGCCGCCATTCAACGGGAGCATAA

ATGGCTTCTATGACCGGTCATCACCATCACCATCACATGGCCAGT AGTGAAGACGTTATCAAGGAGTTTATGCGTTTCAAAGTACGTATG GAGGGTAGTGTTAACGGACACGAATTTGAGATCGAGGGAGAGGGG GAAGGTCGTCCTTACGAGGGAACTCAAACGGCCAAATTAAAGGTG ACCAAAGGTGGGCCCTTGCCATTCGCGTGGGACATCTTGTCACCC CAGTTCCAGTACGGGTCGAAGGCATACGTAAAACACCCAGCGGAC ATTCCTGACTATCTTAAGTTATCTTTCCCGGAAGGTTTTAAATGG GAACGCGTGATGAACTTTGAGGATGGGGGGGTTGTTACGGTGACA CAAGACTCCTCATTGCAAGATGGAGAGTTTATCTATAAAGTCAAA CTTCGCGGCACCAATTTTCCATCTGACGGTCCTGTAATGCAGAAA AAAACAATGGGCTGGGAAGCCTCCACAGAACGTATGTACCCCGAA GATGGAGCTTTAAAGGGCGAAATTAAAATGCGCTTAAAACTTAAA GACGGCGGCCATTACGACGCCGAAGTGAAAACGACGTATATGGCT AAGAAACCCGTCCAGCTTCCGGGAGCCTATAAAACTGACATCAAA CTGGATATTACATCACACAACGAAGATTATACTATTGTCGAACAG TACGAACGCGCCGAAGGCCGCCATTCAACGGGATAGGGCAAACGC AAAAGCTAGTAA
Amino acid sequence ${ }^{\mathrm{a}}$ MVVKFTDSQIQHLMEYGDN DWSEAEFEDAAARDKEFSS QFSKLKSANDKGLKDVIAN PRNDLTDLENKIREKLAAR GFIEVHTPIFVSKSALAKM TITEDHPLFKQVFWIDDKR ALRPMHAMNLYKVMRELRD HTKGPVKIFEIGSCFRKES KSSTHLEEFTMLNLVEMGP DGDPMEHLKMYIGDIMDAV GVEYTTSREESDVYVETLD VEINGTEVASGAVGPHKLD PAHDVHEPWAGIGFGLERL LMLKNGKSNARKTGKSITY LNGYKLD

MASMTGHHHHHHXASSEDV IKEFMRFKVRMEGSVNGHE FEIEGEGEGRPYEGTQTAK LKVTKGGPLPFAWDILSPQ FQYGSKAYVKHPADIPDYL KLSFPEGFKWERVMNFEDG GVVTVTQDSSLQDGEFIYK VKLRGTNFPSDGPVMQKKT MGWEASTERMYPEDGALKG EIKMRLKLKDGGHYDAEVK TTYMAKKPVQLPGAYKTDI KLDITSHNEDYTIVEQYER AEGRHSTGA

MASMTGHHHHHHMASSEDV IKEFMRFKVRMEGSVNGHE FEIEGEGEGRPYEGTQTAK LKVTKGGPLPFAWDILSPQ FQYGSKAYVKHPADIPDYL KLSFPEGFKWERVMNFEDG GVVTVTQDSSLQDGEFIYK VKLRGTNFPSDGPVMQKKT MGWEASTERMYPEDGALKG EIKMRLKLKDGGHYDAEVK TTYMAKKPVOLPGAYKTDI KLDITSHNEDYTIVEQYER AEGRHSTGXGKRKSX 
RFP 204X/237X ATGGCTTCTATGACCGGTCATCACCATCACCATCACATGGCCAGT AGTGAAGACGTTATCAAGGAGTTTATGCGTTTCAAAGTACGTATG GAGGGTAGTGTTAACGGACACGAATTTGAGATCGAGGGAGAGGGG GAAGGTCGTCCTTACGAGGGAACTCAAACGGCCAAATTAAAGGTG ACCAAAGGTGGGCCCTTGCCATTCGCGTGGGACATCTTGTCACCC CAGTTCCAGTACGGGTCGAAGGCATACGTAAAACACCCAGCGGAC ATTCCTGACTATCTTAAGTTATCTTTCCCGGAAGGTTTTAAATGG GAACGCGTGATGAACTTTGAGGATGGGGGGGTTGTTACGGTGACA CAAGACTCCTCATTGCAAGATGGAGAGTTTATCTATAAAGTCAAA CTTCGCGGCACCAATTTTCCATCTGACGGTCCTGTAATGCAGAAA AAAACAATGGGCTGGGAAGCCTCCACAGAACGTATGTACCCCGAA GATGGAGCTTTAAAGGGCGAAATTAAAATGCGCTTAAAACTTAAA GACGGCGGCCATTACGACGCCGAAGTGAAAACGACGTATATGGCT AAGAAACCCGTCCAGCTTCCGGGATAGTATAAAACTGACATCAAA CTGGATATTACATCACACAACGAAGATTATACTATTGTCGAACAG TACGAACGCGCCGAAGGCCGCCATTCAACGGGATAGTAA

\begin{tabular}{ll}
\hline GB1 11X & ATGGCTTCTATGACCGGTATGACCTACAAACTGATCCTGAACGGT \\
& AAATAGCTGAAAGGTGAAACCACCACCGAAGCGTTGACGCGGCG \\
& ACCGCGGAAAAGTTTTCAAACAGTACGCGAACGACAACGGTGTT \\
& GACGGTGAATGGACCTACGACGACGCGACCAAAACCTTCACCGTT \\
& ACCGAAGAAACCTGTATTTTCAGGGCCATCATCATCACCATCAC \\
& TAA
\end{tabular}

GB1 24X/28X ATGGCTTCTATGACCGGTATGACCTACAAACTGATCCTGAACGGT AAAACCCTGAAAGGTGAAACCACCACCGAAGCGGTTGACGCGTAG ACCGCGGAATAGGTTTTCAAACAGTACGCGAACGACAACGGTGTT GACGGTGAATGGACCTACGACGACGCGACCAAAACCTTCACCGTT ACCGAAGAAAACCTGTATTTTCAGGGCCATCATCATCACCATCAC TAA

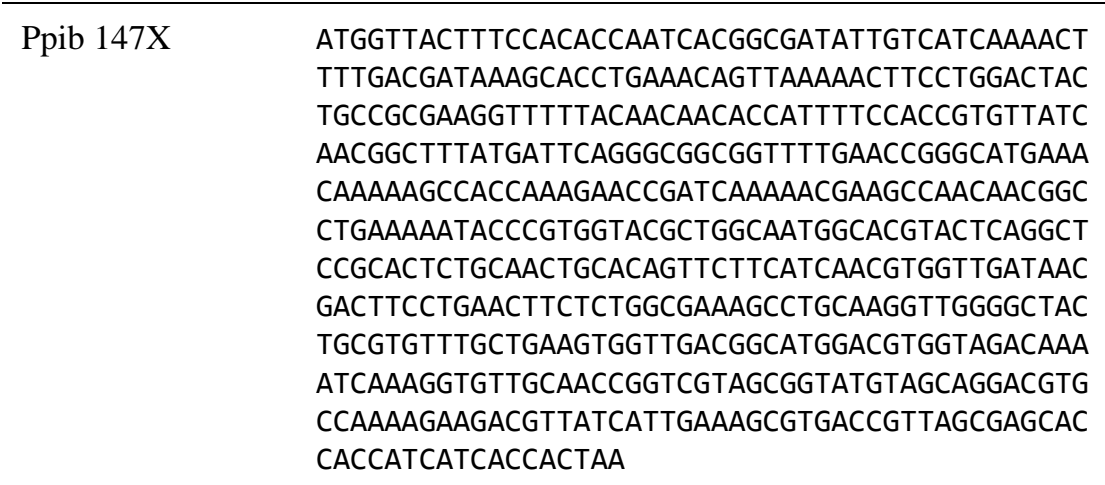

NT-Ubi $7 X^{\mathrm{b}} \quad$ ATGAGCCATACCACACCGTGGACCAATCCTGGTCTGGCAGAAAAC TTTATGAATAGCTTTATGCAGGGTCTGAGCAGCATGCCTGGTTTT ACCGCAAGCCAGCTGGACAAAATGAGCACCATTGCACAGAGCATG GTTCAGAGCATTCAGAGCCTGGCAGCACAGGGTCGTACCAGTCCG AATGATCTGCAGGCACTGAATATGGCATTTGCAAGCAGCATGGCA GAAATTGCAGCAAGCGAAGAAGGTGGCGGTAGCCTGAGCACCAAA ACCAGCAGCATTGCAAGCGCAATGAGCAATGCATTTCTGCAGACA ACCGGTGTTGTTAATCAGCCGTTTATTAACGAAATTACCCAGCTG GTTAGCATGTTTGCACAGGCAGGTATGAATGATGTTAGCGCAGGT AATAGCGGTGGTGGTGGTAGCGGTGAAAACCTGTATTTTCAGTGC GGCAAACGCAAAAGCTAGGGCGGTGGTGGCAGCATGCAGATCTTC GTGAAGACTCTGACTGGTAAGACCATCACCCTCGAGGTTGAGCCC AGTGACACCATTGAGAATGTCAAGGCAAAGATCCAAGATAAGGAA GGCATCCCTCCTGACCAGCAGAGGCTGATCTTTGCTGGAAAACAG CTGGAAGATGGGCGCACCCTGTCTGACTACAACATCCAGAAAGAG TCCACCCTGCACCTGGTACTCCGTCCGAGAGGTGGACACCATCAT CACCACCATTAA
MASMTGHHHHHHMASSEDV IKEFMRFKVRMEGSVNGHE FEIEGEGEGRPYEGTQTAK LKVTKGGPLPFAWDILSPQ FQYGSKAYVKHPADIPDYL KLSFPEGFKWERVMNFEDG GVVTVTQDSSLQDGEFIYK VKLRGTNFPSDGPVMQKKT MGWEASTERMYPEDGALKG EIKMRLKLKDGGHYDAEVK TTYMAKKPVQLPGXYKTDI KLDITSHNEDYTIVEQYER AEGRHSTGX

MASMTGMTYKLILNGKXLK GETTTEAVDAATAEKVFKQ YANDNGVDGEWTYDDATKT FTVTEENLYFQGHHHHHH

MASMTGMTYKLILNGKTLK GETTTEAVDAXTAEXVFKQ YANDNGVDGEWTYDDATKT FTVTEENLYFQGHHHHHH

MVTFHTNHGDIVIKTFDDK APETVKNFLDYCREGFYNN TIFHRVINGFMIQGGGFEP GMKQKATKEPIKNEANNGL KNTRGTLAMARTQAPHSAT AQFFINVVDNDFLNFSGES LQGWGYCVFAEVVDGMDVV DKIKGVATGRSGMXQDVPK EDVIIESVTVSEHHHHHH

MSHTTPWTNPGLAENFMNS FMQGLSSMPGFTASQLDKM STIAQSMVQSIQSLAAQGR TSPNDLQALNMAFASSMAE IAASEEGGGSLSTKTSSIA SAMSNAFLQTTGVVNQPFI NEITQLVSMFAQAGMNDVS AGNSGGGGSGENLYFQCGK RKSXGGGGSMQIFVKTLTG KTITLEVEPSDTIENVKAK IQDKEGIPPDQQRLIFAGK QLEDGRTLSDYNIQKESTL HLVLRPRGGHHHHHH 


\section{$\mathrm{G}^{\mathrm{m} C N P R S^{\mathrm{c}}}$}

\begin{abstract}
ATGCACCACCACCACCACCATGAGAATCTGTATTTCCAGGGTATG GTGGTGAAATTTACCGATAGCCAGATTCAGCATCTGATGGAATAT GGTGATAATGATTGGAGCGAAGCCGAATTTGAAGATGCAGCAGCA CGTGATAAAGAATTTAGCAGCCAGTTTAGCAAACTGAAAAGCGCC AATGATAAAGGCCTGAAAGATGTTATTGCAAATCCGCGTAATGAT CTGACCGATCTGGAAAACAAAATTCGCGAAAAACTGGCAGCCCGT GGTTTTATTGAAGTTCATACCCCGATTTTTGTGAGCAAAAGCGCA CTGGCAAAAATGACCATTACCGAAGATCATCCGCTGTTCAAACAG GTGTTTTGGATTGATGATAAACGTGCACTGCGTCCGATGCATGCA ATGAATGCTTTGAAAGTTATGCGTGAACTGCGCGATCATACCAAA GGTCCGGTTAAAATCTTTGAAATTGGTAGCTGCTTTCGCAAAGAA AGCAAAAGCAGTACCCATCTGGAAGAATTTACCATGCTGAACCTG GCAGAAATGGGTCCTGATGGTGATCCGATGGAACATCTGAAAATG TATATTGGCGATATCATGGATGCCGTTGGTGTTGAATATACCACC AGTCGTGAAGAATCAGATGTTTGGGTTGAAACCCTGGACGTGGAA ATTAATGGCACCGAAGTTGCAAGCGGTTCTGTTGGTCCGCATAAA CTGGATCCGGCACATGATGTGCATGAACCGTGGGCAGGTATTGGT TTTGGTCTGGAACGTCTGCTGATGCTGAAAAATGGTAAAAGCAAT GCACGCAAAACCGGCAAAAGTATTACCTATCTGAATGGCTACAAA CTGGATTAA
\end{abstract}

MHHHHHHENLYFOGMVVKF TDSQIQHLMEYGDNDWSEA EFEDAAARDKEFSSQFSKL KSANDKGLKDVIANPRNDL TDLENKIREKLAARGFIEV HTPIFVSKSALAKMTITED HPLFKQVFWIDDKRALRPM HAMNALKVMRELRDHTKGP VKIFEIGSCFRKESKSSTH LEEFTMLNLAEMGPDGDPM EHLKMYIGDIMDAVGVEYT TSREESDVWVETLDVEING TEVASGSVGPHKLDPAHDV HEPWAGIGFGLERLLMLKN GKSNARKTGKSITYLNGYK $\underline{\mathrm{LD}}$

${ }^{a} \mathrm{X}$ identifies the positions of the ncAA.

${ }^{\mathrm{b}}$ The amino acid sequence of Cys-Ubi $7 \mathrm{X}$ is underlined.

${ }^{c}$ Sequence of G1mCNPRS with the N-terminal His 6 tag and TEV recognition sequence. The amino acid sequence of G1mCNPRS after TEV cleavage is underlined. 
Table S7. List of primers used in the present study.

Primer DNA sequence

\begin{tabular}{|c|c|}
\hline T7 Promotor Forward Primer & TAATACGACTCACTATAGGG \\
\hline T7 Promotor Reverse Primer & CCCTATAGTGAGTCGTATTA \\
\hline T7 Terminator Forward Primer & CCGCTGAGCAATAACTAGC \\
\hline T7 Terminator Reverse Primer & GCTAGTTATTGCTCAGCGG \\
\hline G1RSlib-frag1 Forward Primer & TCATACGCCGTTATACGTTGT \\
\hline G1RSlib-frag1 Reverse Primer & TTCATTGCATGCATCGGACG \\
\hline G1RSlib-frag2 Forward Primer & $\begin{array}{l}\text { CGATGCATGCAATGAATNNKNNKAAAGTTATGCGTGAA } \\
\text { CTGCGC }\end{array}$ \\
\hline G1RSlib-frag2 Reverse Primer & $\begin{array}{l}\text { CCATCAGGACCCATTTCKVMCAGGNYCAGCATGGTAAA } \\
\text { TTCTTCCAGATG }\end{array}$ \\
\hline G1RSlib-frag3 Forward Primer & AAATGGGTCCTGATGGTGATC \\
\hline G1RSlib-frag3 Reverse Primer & CGCTTGCAACTTCGGTG \\
\hline G1RSlib-frag4 Forward Primer & $\begin{array}{l}\text { ACCGAAGTTGCAAGCGGTNNKGTTGGTCCGCATAAACT } \\
\text { GG }\end{array}$ \\
\hline G1RSlib-frag4 Reverse Primer & $\begin{array}{l}\text { GACCAAAACCAATACCTGCMNNCGGTTCATGCACATCA } \\
\text { TGTG }\end{array}$ \\
\hline G1RSlib-frag5 Forward Primer & CAGGTATTGGTTTTGGTCTGGAAC \\
\hline G1RSlib-frag5 Reverse Primer & GCGAAGAAAAGCAGAAAAAACG \\
\hline G1RSY204F Forward Primer & TCAGATGTTTTTGTTGAAACCCTGGACGTGG \\
\hline G1RSY204F Reverse Primer & GTTTCAACAAAAACATCTGATTCTTCACGACTG \\
\hline G1RSY204W Forward Primer & TCAGATGTTTGGGTTGAAACCCTGGACGTGG \\
\hline G1RSY204W Reverse Primer & GTTTCAACCCAAACATCTGATTCTTCACGACTG \\
\hline
\end{tabular}


Table S8. Structural data of the single-crystal X-ray structure determined of G1mCNPRS.

\begin{tabular}{|c|c|}
\hline \multicolumn{2}{|c|}{ G1mCNPRS (G1PylRS mutant - G1mCNP34) } \\
\hline PDB ID & $7 \mathrm{R} 6 \mathrm{O}$ \\
\hline \multicolumn{2}{|l|}{ Data collection } \\
\hline Space group & $\mathrm{P} 12_{1} 1$ \\
\hline \multicolumn{2}{|l|}{ Cell dimensions } \\
\hline$a, b, c(\AA)$ & $58.9,81.4,121.9$ \\
\hline$\alpha, \beta, \gamma\left(^{\circ}\right)$ & $90,102.1,90$ \\
\hline Resolution $(\AA)$ & $48.06-2.2(2.279-2.2)^{*}$ \\
\hline$R_{\text {merge }}$ & $0.16(2.31)$ \\
\hline$R_{\text {pim }}$ & $0.063(0.93)$ \\
\hline $\mathrm{I} / \sigma \mathrm{I}$ & $6.7(0.9)$ \\
\hline $\mathrm{CC}_{1 / 2}$ & $0.998(0.341)$ \\
\hline Completeness (\%) & $99.9(99.9)$ \\
\hline Multiplicity & $7.0(7.1)$ \\
\hline \multicolumn{2}{|l|}{ Refinement } \\
\hline Resolution $(\AA)$ & $46.56-2.2(2.279-2.2)$ \\
\hline No. reflections & 57135 \\
\hline$R_{\text {work }} / R_{\text {free }}$ & $0.2187 / 0.2684(0.3473 / 0.3102)$ \\
\hline No. atoms & 9301 \\
\hline Protein & 8660 \\
\hline Ligand/ion & 17 \\
\hline Water & 624 \\
\hline B-factors (overall) & 56.06 \\
\hline Protein & 56.38 \\
\hline Ligand/ion & 77.34 \\
\hline Water & 51.04 \\
\hline \multicolumn{2}{|l|}{ R.m.s. deviations } \\
\hline Bond lengths $(\AA)$ & 0.002 \\
\hline Bond angles $\left({ }^{\circ}\right)$ & 0.47 \\
\hline
\end{tabular}

* Statistics for the highest resolution shell are shown in parentheses. 


\section{Synthesis of EDDC, and mCNP}

Reactions were conducted under a positive pressure of dry nitrogen in oven-dried glassware and at room temperature, unless specified otherwise. Starting materials, solvents, and reagents were purchased from commercial sources and used as such for the reactions. Analytical thin-layer chromatography was conducted with aluminium-backed silica gel $60 \mathrm{~F}_{254}(0.2 \mathrm{~mm})$ plates supplied by Merck and visualized using UV fluorescence $\left(\lambda_{\max }=254 \mathrm{~nm}\right)$, or developed using basic $\mathrm{KMnO}_{4}$ solution followed by heating. For filtration column chromatography, Merck Kiesegel 60 silica gel (230 - 400 mesh) was used. Flash chromatography was conducted on a Biotage Isolera One.

${ }^{1} \mathrm{H}-\mathrm{NMR}$ spectra were recorded at $400 \mathrm{MHz}$ using a Bruker Avance $400 \mathrm{MHz} \mathrm{NMR}$ spectrometer. Residual solvent peaks were used as an internal reference for ${ }^{1} \mathrm{H}-\mathrm{NMR}$ spectra $\left(\mathrm{D}_{2} \mathrm{O} \delta=4.79\right)$. Coupling constants $(J)$ are quoted to the nearest $0.1 \mathrm{~Hz}$. The assignment of proton signals was assisted by COSY, HSQC, and HMBC experiments. ${ }^{13} \mathrm{C}$-NMR spectra were recorded at $100 \mathrm{MHz}$ using a Bruker Avance $400 \mathrm{MHz}$ NMR spectrometer. The following abbreviations are used to denote ${ }^{1} \mathrm{H}-\mathrm{NMR}$ multiplicities: $\mathrm{s}=$ singlet, $\mathrm{d}=$ doublet, $\mathrm{t}=$ triplet, $\mathrm{m}=$ multiplet. Low-resolution ESI mass spectra were recorded on a ZMD Micromass spectrometer with Waters Alliance 2690 HPLC. High resolution ESI mass spectra were recorded on a Waters LCT Premier time-of-flight (TOF) mass spectrometer. 


\section{Synthesis of EDDC (ethylenediamine dicysteine)}

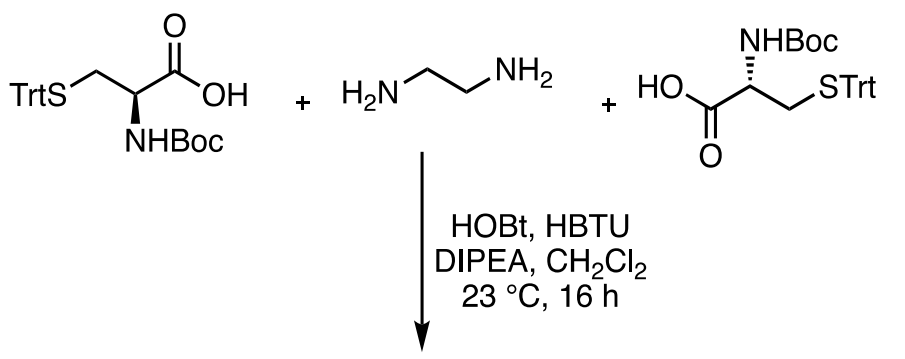

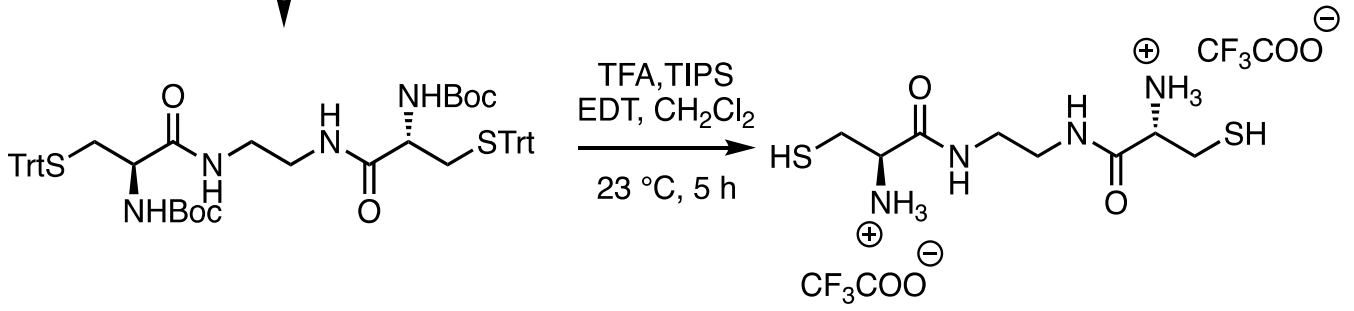

To a solution of Boc-Cys(Trt)-OH ( $3.86 \mathrm{~g}, 8.32 \mathrm{mmol}, 2.0 \mathrm{~mol}$ equiv), HBTU (4.70 g , $12.5 \mathrm{mmol}, 3.0 \mathrm{~mol}$ equiv), HOBt (562 mg, $4.15 \mathrm{~mol}$ equiv, $1.0 \mathrm{~mol}$ equiv), and DIPEA (2.20 mL, $12.5 \mathrm{mmol}, 3.0 \mathrm{~mol}$ equiv) in $\mathrm{CH}_{2} \mathrm{Cl}_{2}(45 \mathrm{~mL})$ was added a solution of ethylenediamine (250 mg, $4.16 \mathrm{mmol}, 1.0 \mathrm{~mol}$ equiv) in $\mathrm{CH}_{2} \mathrm{Cl}_{2}(5 \mathrm{~mL})$ at $0{ }^{\circ} \mathrm{C}$. The reaction mixture was brought to $25{ }^{\circ} \mathrm{C}$ and stirred for $16 \mathrm{~h}$. Reaction completion was confirmed by TLC and mass spectrometric analysis. The reaction mixture was quenched with ice-cold water $(100 \mathrm{~mL})$ and layers were separated. The aqueous layer was extracted with $\mathrm{CH}_{2} \mathrm{Cl}_{2}(30 \mathrm{~mL})$. The combined organic layer was washed with brine solution (20 $\mathrm{mL}$ ), separated, dried over $\mathrm{Na}_{2} \mathrm{SO}_{4}$, filtered, and concentrated under reduced pressure. The crude product was dissolved in $\mathrm{CH}_{2} \mathrm{Cl}_{2}(20 \mathrm{~mL})$, passed through a plug of silica to remove the polar impurities, and washed with $10 \% \mathrm{MeOH}$ in $\mathrm{CH}_{2} \mathrm{Cl}_{2}(50 \mathrm{~mL})$. The solvent was evaporated to obtain the protected ethylendiamine dicysteine as a viscous liquid and used as such for the next step.

The crude product from the coupling reaction was dissolved in $\mathrm{CH}_{2} \mathrm{Cl}_{2}(20 \mathrm{~mL})$ and cooled to $0{ }^{\circ} \mathrm{C}$ before a solution of TFA: TIPS: EDT: water $(10 \mathrm{~mL}: 0.5 \mathrm{~mL}: 0.5 \mathrm{~mL}$ : $0.5 \mathrm{~mL}$ ) was added. The reaction mixture was allowed to warm to $23{ }^{\circ} \mathrm{C}$ and stirred for 5 h. Reaction completion was confirmed by mass spectrometric analysis. The solvent and 
excess reagents were removed by evaporation under reduced pressure. The crude waxy liquid was then triturated with hexane $(2 \times 5 \mathrm{~mL})$ to remove the non-polar impurities and further trituration with $\mathrm{Et}_{2} \mathrm{O}(2 \times 5 \mathrm{~mL})$ yielded the TFA salt of the product as a white amorphous solid (1.45 g, $2.93 \mathrm{mmol}, 70 \%$ over two steps).

${ }^{1} \mathbf{H}$ NMR $\left(400 \mathrm{MHz}, \mathrm{D}_{2} \mathrm{O}\right) \delta 8.51$ (brs, $\left.2 \times \mathrm{CONH}\right), 4.17(\mathrm{t}, J=5.7 \mathrm{~Hz}, 2 \mathrm{H}), 3.55-3.33$ (m, 4H), $3.15-2.99(\mathrm{~m}, 4 \mathrm{H}) \mathrm{ppm}, 1.35(\mathrm{~s}, 2 \times \mathrm{SH}) \mathrm{ppm} ;{ }^{13} \mathbf{C ~ N M R}\left(100 \mathrm{MHz}, \mathrm{D}_{2} \mathrm{O}\right) \quad \delta$ $168.1(2 \times \mathrm{Cq}), 54.5(2 \times \mathrm{CH}), 38.8\left(2 \times \mathrm{CH}_{2}\right), 24.8\left(2 \times \mathrm{CH}_{2}\right) \mathrm{ppm}$; LRMS (ESI+): m/z (\%): $267([\mathrm{M}+\mathrm{H}], 85), 533([2 \mathrm{M}+\mathrm{H}], 80), 555([2 \mathrm{M}+\mathrm{Na}], 100) ; \mathbf{H R}-\mathbf{M S}(\mathrm{ESI}+): \mathrm{m} / \mathrm{z}$ calculated for $\mathrm{C}_{8} \mathrm{H}_{19} \mathrm{~N}_{4} \mathrm{O}_{2} \mathrm{~S}_{2}$ : 267.0949; found: 267.0952 . 


\section{Synthesis of mCNP (L-3-(2-cyano-4-pyridyl)alanine)}
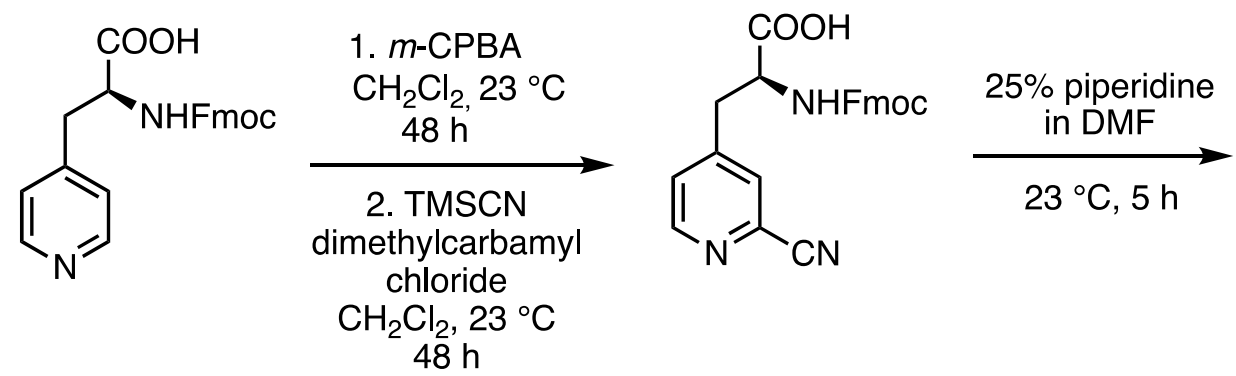<smiles>N#Cc1cc(CC(N)C(=O)O)ccn1</smiles>

Fmoc-L-3-(2-cyano-4-pyridyl)alanine was synthesized according to a reported procedure. ${ }^{15}$ Fmoc-L-3-(2-cyano-4-pyridyl)alanine (1.5 g, $\left.3.63 \mathrm{mmol}\right)$ was solved in 15 $\mathrm{mL}$ of $25 \%$ piperidine solution in DMF and stirred at $23{ }^{\circ} \mathrm{C}$ for $5 \mathrm{~h}$. Reaction completion was confirmed by TLC and mass spectrometric analysis. Excess reagents were removed under reduced pressure and the remaining viscous liquid was stirred with hexane $(2 \times 20$ $\mathrm{mL})$ to remove the non-polar impurities. Upon trituration with diethyl ether $(2 \times 15 \mathrm{~mL})$, pure L-3-(2-cyano-4-pyridyl)alanine was obtained as a white solid (550 mg, $2.88 \mathrm{mmol}$, $79 \%)$

${ }^{1} \mathbf{H}$ NMR $\left(400 \mathrm{MHz}, \mathrm{D}_{2} \mathrm{O}\right) \delta 8.65(\mathrm{~d}, J=4.6 \mathrm{~Hz}, 1 \mathrm{H}), 7.89(\mathrm{~s}, 1 \mathrm{H}), 7.67(\mathrm{~d}, J=3.9 \mathrm{~Hz}$ 1H), $4.05(\mathrm{t}, J=5.9 \mathrm{~Hz}, 1 \mathrm{H}), 3.45-3.17(\mathrm{~m}, 2 \mathrm{H}) \mathrm{ppm} ;{ }^{13} \mathbf{C} \mathbf{N M R}\left(100 \mathrm{MHz}, \mathrm{D}_{2} \mathrm{O}\right) \delta$ $172.9(\mathrm{Cq}), 150.9(\mathrm{CH}), 147.8(\mathrm{Cq}), 132.3(\mathrm{Cq}), 130.2(\mathrm{CH}), 129.0(\mathrm{CH}), 117.0(\mathrm{Cq}), 54.8$ (CH), $35.7\left(\mathrm{CH}_{2}\right)$ ppm; LRMS (ESI-): m/z (\%): 190 ([M-H], 100), 381 ([2M-H], 80); HR-MS (ESI-): m/z calculated for $\mathrm{C}_{9} \mathrm{H}_{8} \mathrm{~N}_{3} \mathrm{O}_{2}: 190.0617$; found: 190.0618 . 


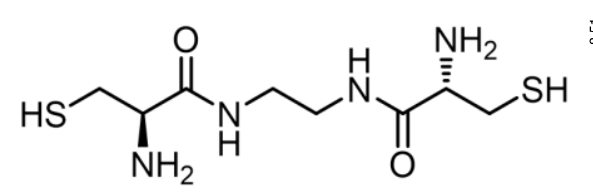

$2 \mathrm{CF}_{3} \mathrm{COOH}$

${ }^{1} \mathrm{H}$ NMR

$400 \mathrm{MHz}, \mathrm{D}_{2} \mathrm{O}$

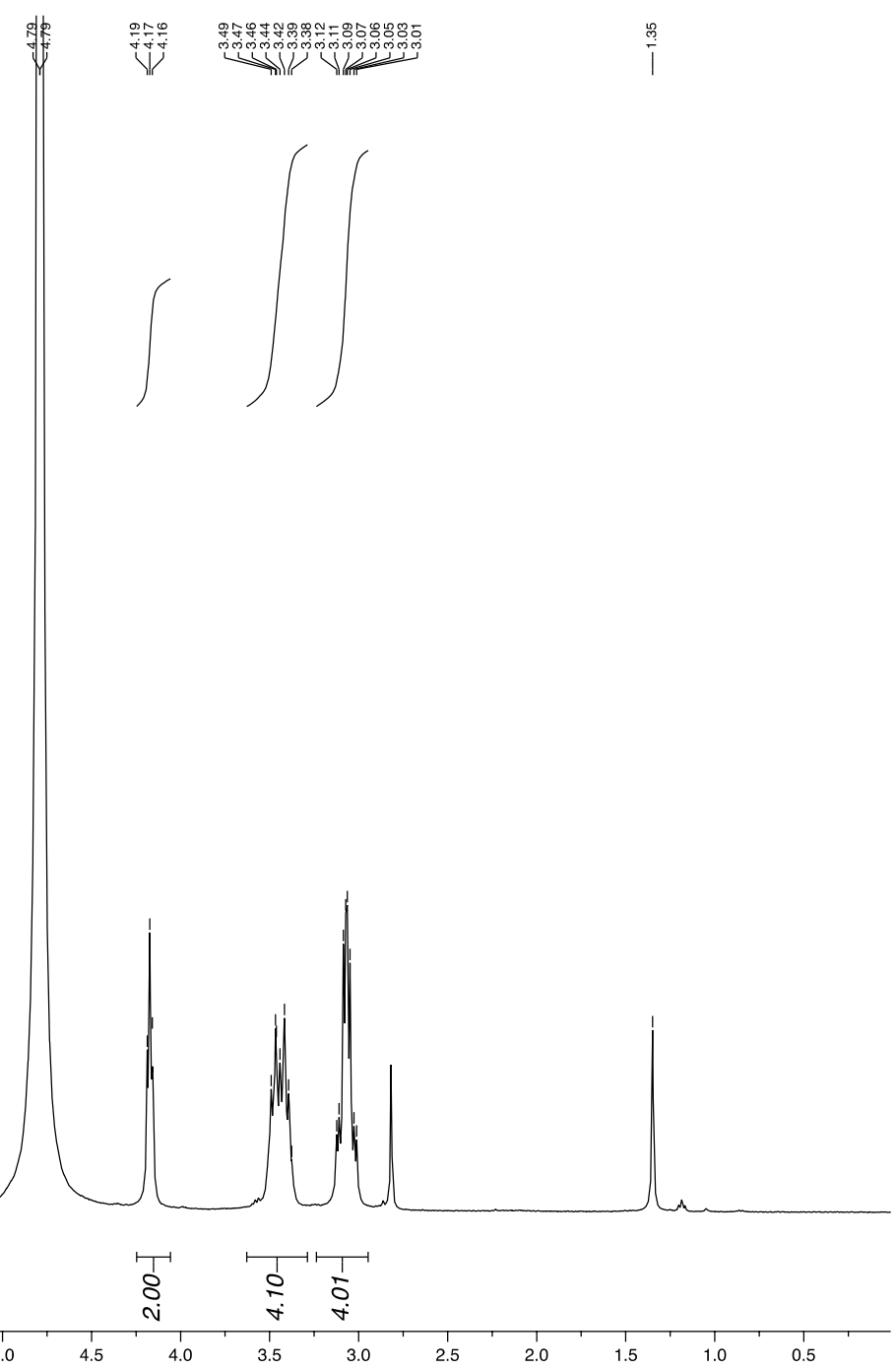


<smiles>NC(CS)C(=O)NCCNC(=O)[C@H](N)CS</smiles>

$2 \mathrm{CF}_{3} \mathrm{COOH}$

${ }^{13} \mathrm{C}$ NMR

$100 \mathrm{MHz}, \mathrm{D}_{2} \mathrm{O}$

.

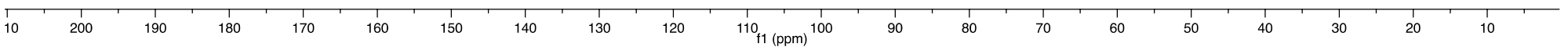




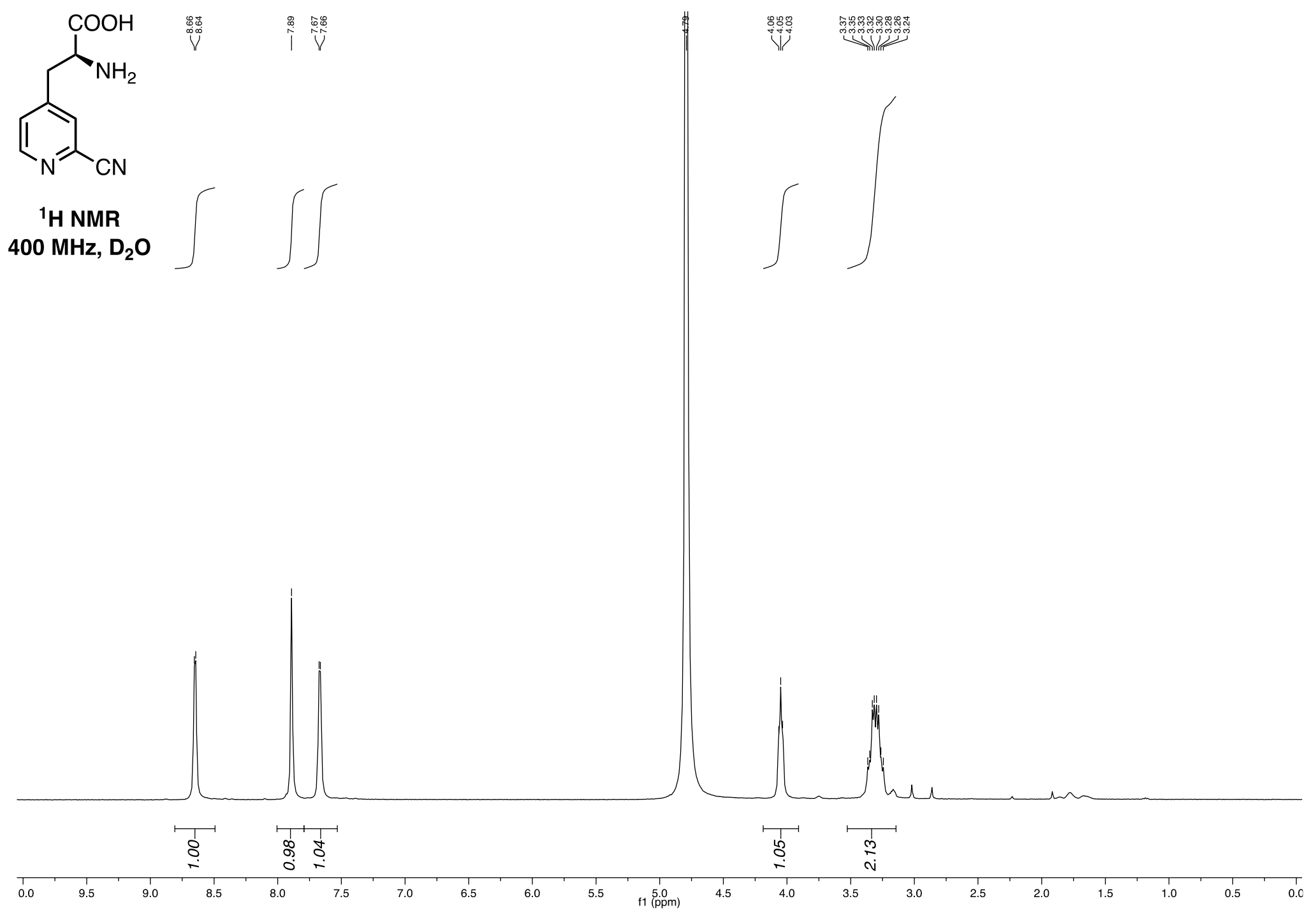

S39 
<smiles>N#Cc1cc(C[C@H](N)C(=O)O)ccn1</smiles>

${ }^{13} \mathrm{C}$ NMR

$400 \mathrm{MHz}, \mathrm{D}_{2} \mathrm{O}$

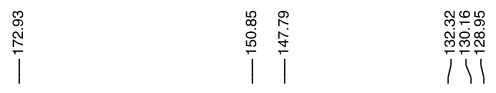

$\stackrel{\cong}{=}$

|

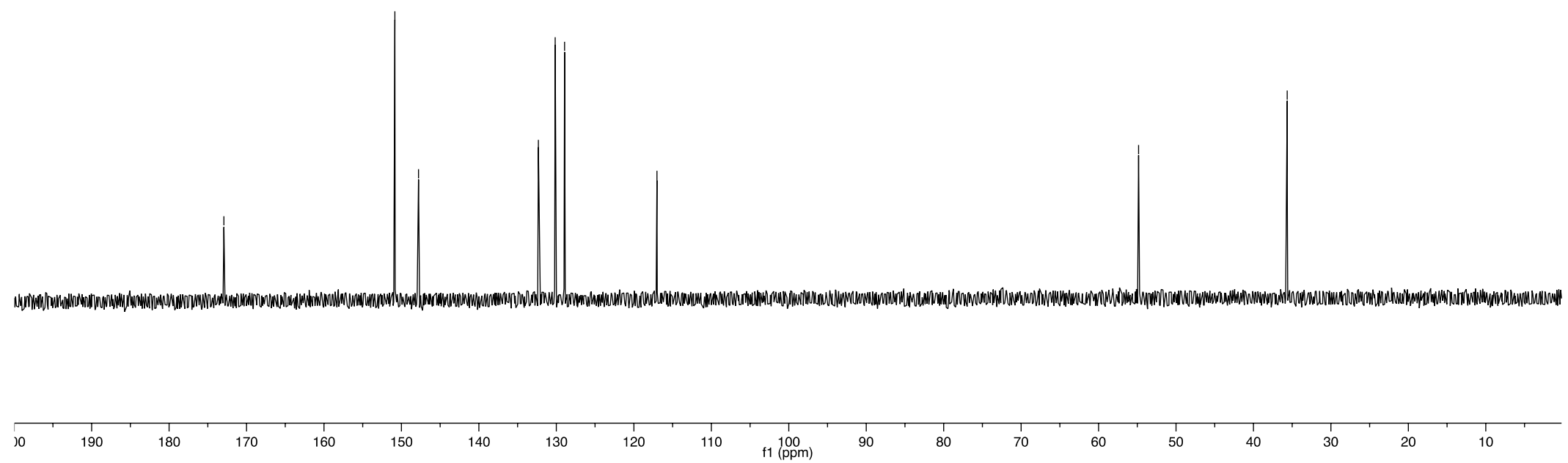

S40 


\section{References}

1. Young, T. S.; Ahmad, I.; Yin, J. A.; Schultz, P. G. An enhanced system for unnatural amino acid mutagenesis in E. coli. J. Mol. Biol. 2010, 395, 361-374.

2. Qianzhu, H.; Welegedara, A. P.; Williamson, H.; McGrath, A. E.; Mahawaththa, M. C.; Dixon, N. E.; Otting, G.; Huber, T. Genetic encoding of para-pentafluorosulfanyl phenylalanine: a highly hydrophobic and strongly electronegative group for stable protein interactions. J. Am. Chem. Soc. 2020, 142, 17277-17281.

3. Abdelkader, E. H.; Qianzhu, H.; Tan, Y. J.; Adams, L. A.; Huber, T.; Otting, G. Genetic Encoding of $N^{6}$-(((Trimethylsilyl)methoxy)carbonyl)-L-lysine for NMR studies of protein-protein and protein-ligand interactions. J. Am. Chem. Soc. 2021, 143, 1133-1143. 4. Mukai, T.; Hoshi, H.; Ohtake, K.; Takahashi, M.; Yamaguchi, A.; Hayashi, A.; Yokoyama, S.; Sakamoto, K. Highly reproductive Escherichia coli cells with no specific assignment to the UAG codon. Sci. Rep. 2015, 5, 1-9.

5. Klopp, J.; Winterhalter, A.; Gébleux, R.; Scherer-Becker, D.; Ostermeier, C.; Gossert, A. D. Cost-effective large-scale expression of proteins for NMR studies. J. Biomol. NMR 2018, 71, 247-262.

6. Aragao, D.; Aishima, J.; Cherukuvada, H.; Clarken, R.; Clift, M.; Cowieson, N. P.; Ericsson, D. J.; Gee, C. L.; Macedo, S.; Mudie, N.; Panjikar, S.; Price, J. R.; RiboldiTunnicliffe, A.; Rostan, R.; Williamson, R.; Caradoc-Davies, T. T. MX2: a high-flux undulator microfocus beamline serving both the chemical and macromolecular crystallography communities at the Australian Synchrotron. J. Synchrotron Radiat. 2018, 25, $885-891$.

7. Kabsch, W. XDS. ACTA Crystallogr. D 2010, 66, 125-132.

8. Evans, P. R.; Murshudov, G. N. How good are my data and what is the resolution? ACTA Crystallogr. D 2013, 69, 1204-1214. 
9. McCoy, A. J.; Grosse-Kunstleve, R. W.; Adams, P. D.; Winn, M. D.; Storoni, L. C.; Read, R. J. Phaser crystallographic software. J. Appl. Crystallogr. 2007, 40, 658-674.

10. Bunkoczi, G.; Read, R. J. Improvement of molecular-replacement models with Sculptor. ACTA Crystallogr. D 2011, 67, 303-312.

11. Emsley, P.; Lohkamp, B.; Scott, W. G.; Cowtan, K. Features and development of Coot. ACTA Crystallogr. D 2010, 66, 486-501.

12. Afonine, P. V.; Grosse-Kunstleve, R. W.; Echols, N.; Headd, J. J.; Moriarty, N. W.; Mustyakimov, M.; Terwilliger, T. C.; Urzhumtsev, A.; Zwart, P. H.; Adams, P. D. Towards automated crystallographic structure refinement with phenix.refine. ACTA Crystallogr. D 2012, 68, 352-367.

13. Zwart, P. H.; Afonine, P. V.; Grosse-Kunstleve, R. W.; Hung, L.-W.; Ioerger, T. R.; McCoy, A. J.; McKee, E.; Moriarty, N. W.; Read, R. J.; Sacchettini, J. C., Automated structure solution with the PHENIX suite. In Structural Proteomics, Springer: 2008; pp 419435 .

14. Kavran, J. M.; Gundllapalli, S.; O'Donoghue, P.; Englert, M.; Söll, D.; Steitz, T. A. Structure of pyrrolysyl-tRNA synthetase, an archaeal enzyme for genetic code innovation. Proc. Natl. Acad. Sci. U.S.A. 2007, 104, 11268-11273.

15. Nitsche, C.; Onagi, H.; Quek, J.-P.; Otting, G.; Luo, D.; Huber, T. Biocompatible macrocyclization between cysteine and 2-cyanopyridine generates stable peptide inhibitors. Org. Lett. 2019, 21, 4709-4712. 